\title{
upf. $\begin{array}{ll}\text { Universitat } & \text { Department } \\ \text { Pompeu Fabra } & \text { of Economics and Business }\end{array}$ Barcelona
}

Economics Working Paper Series

Working Paper No. 1573

\section{Aggregation and design of information in asset markets with adverse selection}

Vladimir Asriyan, William Fuchs and Brett Green

Updated version: February 2019

(July 2017) 


\title{
Aggregation and Design of Information in Asset Markets with Adverse Selection
}

\author{
Vladimir Asriyan, William Fuchs, and Brett Green*
}

February 14, 2019

\begin{abstract}
How effectively does a decentralized marketplace aggregate information that is dispersed throughout the economy? We study this question in a dynamic setting where sellers have private information that is correlated with an unobservable aggregate state. In any equilibrium, each seller's trading behavior provides an informative and conditionally independent signal about the aggregate state. We ask whether the state is revealed as the number of informed traders grows large. Surprisingly, the answer is no; we provide conditions under which information aggregation necessarily fails. In another region of the parameter space, aggregating and non-aggregating equilibria coexist. We solve for the optimal information policy of a constrained social planner who observes trading behavior and chooses what information to reveal. We show that non-aggregating equilibria are always constrained inefficient. The optimal information policy Pareto improves upon laissez-faire outcomes by concealing favorable information in order to accelerate trade.
\end{abstract}

JEL: G14, G18, D47, D53, D82, D83.

Keywords: Information Aggregation; Information Design; Decentralized Markets; Adverse Selection; Optimal Information Policy; Transparency.

*Asriyan: CREi, UPF, Barcelona GSE and CEPR. Fuchs: UT Austin McCombs School of Business and Universidad Carlos III de Madrid. Green: Haas School of Business, UC Berkeley. Asriyan acknowledges financial support from the Spanish Ministry of Economy and Competitiveness, through the Severo Ochoa Programme for Centres of Excellence in R\&D (SEV-2015-0563). Fuchs acknowledges support from the ERC Grant 681575. We thank Piero Gottardi, Stephan Lauermann (discussant), Victoria Vanasco, Venky Venkateswaran and seminar/conference participants at CREi-UPF, EIEF, EUI, HEC Paris, Imperial College London, TSE, UC3M, AEA meetings in Atlanta, Advances in Information Economics Conference in Como, FTG meetings in London, 17th SAET Conference on Current Trends in Economics, and SED meetings in Edinburgh for helpful discussions and feedback. Joon Sup Park and Mark Whitmeyer provided excellent research assistance. 


\section{Introduction}

Since the seminal work of Hayek (1945), the question of whether markets effectively aggregate dispersed information has been a central one in economics. Formal investigations of this question are plentiful (see Section 1.1 for a discussion). Yet, they are typically conducted in a setting with a single asset about which traders have dispersed information. Whether information is aggregated then usually boils down to whether the equilibrium price reveals the value of the asset conditional on the union of traders' information. This broad class of models is natural for many applications from static common-value auctions to dynamic trading in financial markets. For other applications (e.g., real estate, OTC markets), information dispersion arises due to dispersion in ownership, and one is interested in the extent to which aggregate trading behavior across heterogeneous assets reveals information about the underlying state of the economy. In this paper, we explore such a setting.

Building on the framework of Asriyan, Fuchs, and Green (2017), we investigate the question of information aggregation in a dynamic setting with many assets, whose values are independently and identically drawn from a distribution that depends on an underlying aggregate state. The value of each asset is privately observed by its seller, who receives offers each period from competitive buyers. We ask whether the history of all transactions reveals the aggregate state as the number of informed sellers in the economy (denoted by $N$ ) grows large.

To answer this question, we begin by characterizing the set of equilibria for arbitrary $N$. Due to a complementarity between the amount of information collectively revealed by others and the optimal strategy of an individual seller, multiple equilibria can exist. A feature common to all equilibria is that each individual seller's trading behavior provides an informative and conditionally independent signal about the aggregate state. Therefore, one might intuitively expect that, by the law of large numbers, the state would be revealed as the number of sellers tends to infinity.

Our first main result highlights that this intuition is incomplete because it ignores the sellers' endogenous response to the expectation of information arrival, which in turn changes the information content of each individual trade. We provide necessary and sufficient conditions under which there does not exist a sequence of equilibria that reveal the state as $N \rightarrow \infty$. The reason why aggregation fails is that the information content of each individual seller's behavior tends to zero at a rate of $1 / N$, just fast enough to offset the additional number of observations. As a result, some information is revealed by the limiting trading behavior, but not enough to precisely determine the underlying state. Roughly speaking, the conditions for non-aggregation require that the correlation of asset values is sufficiently high and that agents are sufficiently patient. Intuitively, these conditions guarantee that if the aggregate state were to be revealed 
with certainty tomorrow, then the option value of delaying trade today is relatively high. An immediate corollary is that information aggregation always obtains in a static model. That is, dynamic considerations are a necessary ingredient for non-aggregation.

When the parametric conditions for non-aggregation do not hold, there exists a sequence of equilibria such that information about the state is aggregated as $N \rightarrow \infty$. However, even in this case, information aggregation is not guaranteed. Our second main result shows that there is a region of the parameter space in which there is coexistence of equilibria that reveal the state with equilibria that do not. The key difference across the two types of equilibria is the rate at which trade declines as the number of informed sellers grows. In the non-aggregating equilibria, trade declines at rate $1 / N$ whereas in aggregating equilibria, the rate of trade declines slower than $1 / N$. We are not aware of analogous coexistence results in the literature.

Whether information aggregates has important implications for welfare, prices, and trading behavior. To understand them, it is useful to draw comparison to a fictitious economy in which the state is exogenously revealed after the first trading period. When information aggregates, both trading volume and welfare converge to their levels in the fictitious economy and the volatity of prices conditional on the true state goes to zero. In contrast, along a sequence of non-aggregating equilibria, trading volume and welfare are strictly lower than in the fictitious economy and the conditional price volatility remains strictly positive even as $N \rightarrow \infty$.

Two immediate implications follow. First, from a social welfare perspective, aggregating equilibria are always preferable to non-aggregating equilibria when they co-exist. Thus, among laissez-faire outcomes, aggregation is optimal. Second, if all equilibria are non-aggregating, then a planner could improve overall welfare by learning and revealing the true state. Of course, it is not obvious how the planner could learn the true state. It is more natural to think that the planner is uninformed, but can learn about the true state by observing the trading behavior of market participants. The problem facing the planner is then how best to reveal this information to other agents in the economy. The question we ask is whether an uninformed planner can improve on the laissez-faire outcomes.

We address this question in Section 4. Doing so involves formulating and solving an information design problem related to the literature on Bayesian persuasion Kamenica and Gentzkow, 2011; Rayo and Segal, 2010). One key difference is that the planner's problem in our model must take into account the fact that her policy influences the information content of trading behavior and therefore the information content of whatever is revealed. In other words, the statistical properties of the information that is revealed by the planner endogenously depend on the planner's policy. Our solution technique involves two steps. First, we solve the information design problem for an "informed" planner who (exogenously) learns the aggregate state. The solution to this problem helps us derive an upper bound on the surplus that can be attained 
by an uninformed planner. We then construct a policy under which the payoffs converge to the upper bound as $N \rightarrow \infty$.

Indeed, we demonstrate that when information aggregation fails, the outcome is not constrained efficient. Perhaps counterintuitively, the planner can improve on such outcomes by concealing some information about trading behavior in order to promote more gains from trade being realized. Moreover, information aggregation does not necessarily imply constrained efficiency. Even when information aggregates, the optimal information policy can improve overall efficiency when the discount factor is sufficiently high. Importantly, the optimal information policy Pareto dominates laissez-faire outcomes. These findings have obvious implications for policies aimed at promoting market transparency.

Recently, there has been a strong regulatory push towards making financial markets more transparent (i.e., disclosing more information about trading activity to market participants). For example, one of the stated goals of the Dodd-Frank Act of 2010 is to increase transparency and information dissemination in the financial system. The European Commission is considering revisions to the Markets in Financial Instruments Directive (MiFID), in part to improve the transparency of European financial markets. Our results highlight a potential trade-off for such policies and provide a potential justification for limiting the amount of information available to market participants.

Relatedly, the introduction of benchmarks that reveal some aggregate trading information has also received recent attention by policy makers and academics. Duffie et al. (2017) analyze the role of benchmarks (e.g., LIBOR) in revealing information about fundamentals and suggest that the introduction of benchmarks is welfare enhancing. Our analysis highlights an important consideration that is absent in their setting. Namely, that the informational content of the benchmark may change once it is published due to endogenous responses by market participants.

\subsection{Related Literature}

Within static environments, there is a large literature that studies questions regarding information aggregation. Seminal works on this topic include Grossman (1976), Wilson (1977), Milgrom (1979), Hellwig (1980), and Kyle (1989). More recent progress on this question has been made by Pesendorfer and Swinkels (1997), Kremer (2002), Rostek and Weretka (2012), Lauermann and Wolinsky (2017), Bodoh-Creed (2013), Albagli et al. (2015), Axelson and Makarov (2017), and Siga and Mihm (2018), among others ${ }^{1}$ By and large, this literature is largely defined by a centralized trading environment in which there is a single asset about which agents have dispersed information. The question of information aggregation is whether the price is a sufficient

\footnotetext{
$\sqrt[1]{\text { Palfrey }}(1985)$ and $\operatorname{Vives}(1988)$ explore this question within a Cournot setting.
} 
statistic for the union of this dispersed information. In contrast, we explore a decentralized trading environment with heterogeneous assets and ask whether the history of trading behavior is sufficient to infer the underlying state. Moreover, our results pertaining to non-aggregation crucially rely on dynamic considerations - with a single opportunity to trade, information is always aggregated in our setting.

Kyle (1985) studies a dynamic insider trading model and shows that the insider fully reveals his information as time approaches the end of the trading interval. Foster and Viswanathan (1996) and Back et al. (2000) extend this finding to a model with multiple strategic insiders with different information. Ostrovsky (2012) further generalizes these findings to a broader class of securities and information structures. He considers a dynamic trading model with finitely many partially informed traders and provides necessary and sufficient conditions on security payoffs for information aggregation to obtain. Our paper differs from these works in that we study a setting with heterogeneous but correlated assets owned by privately informed sellers. We ask whether information aggregates as the number of sellers becomes arbitrarily large. Despite the fact that we look at the limit as $N \rightarrow \infty$, the strategic considerations do not vanish in our model since there is an idiosyncratic component to the value of each asset.

Golosov et al. (2014) consider an environment in which some agents have private information about an asset while the rest are uninformed. Agents trade in a decentralized anonymous market through bilateral matches, i.e., signaling with trading histories is not possible. They find that information aggregation obtains in the long run. In contrast, in our setting observing trading histories plays a crucial role: signaling through delay diminishes the amount of trade, thus reducing the information content of the market, leading to the possibility that information aggregation fails.

Lauermann and Wolinsky (2016) study information aggregation in a search market, in which an informed buyer sequentially solicits offers from sellers who have noisy information about the buyer's value. They provide conditions under which information aggregation fails, and they trace this failure to a strong form of winner's curse that arises in a search environment. Although our model is quite different, we share the common feature that the fear of adverse selection hinders trade and thus reduces information generation in markets.

Also, within a search framework, Lester et al. (2018) look at how equilibrium trade, margins and information changes as the probability of meeting a dealer is increased. They show that as the meeting frequency increases the information flow to the market might decrease. Roughly speaking, this corresponds to our finding that information aggregation is more likely to fail as we increase the discount factor $2^{2}$ Relatedly, Maestri et al. (2016) study an asset market with adverse selection and aggregate uncertainty about asset values and show that full efficiency is

\footnotetext{
${ }^{2} \mathrm{~A}$ higher discount factor translates into a lower expected time until the next opportunity to trade.
} 
attained as search frictions vanish. However, their setting does not feature signaling through delay, which is essential for our results. In our setting, trade remains inefficient evan as trading frictions vanish (i.e., $\delta \rightarrow 1$ ).

Babus and Kondor (2016) explore how the network structure affects information diffusion in a static OTC model with a single divisible asset. They show that strategic considerations do not influence the degree of information diffusion. However, the network structure combined with a private value component leads to an informational externality that constrains the informativeness of prices and hence the informational efficiency of the economy.

In addition to the welfare implications studied in this paper, there are a variety of other reasons for why information aggregation may be a desirable property. For instance, such information may be useful for informing firms' investment decisions (Fishman and Hagerty, 1992; Leland, 1992; Dow and Gorton, 1997; Camargo et al., 2015), government interventions (Bond et al. 2009; Bond and Goldstein, 2015; Boleslavsky et al., 2017), and monetary policy (Bernanke and Woodford, 1997). Markets that convey more information can also be more useful for providing better incentives to managers (Baumol, 1965; Fishman and Hagerty, 1989) and mitigating the winner's curse in common-value auctions (Milgrom and Weber, 1982). As documented by a number of papers in this literature, the feedback loop between real decisions and price informativeness may undermine the ability of markets to aggregate information and lead to aggregation failures..$^{3}$ To highlight how our mechanism differs from this literature, we abstract from any such considerations here.

Finally, our paper is related to a growing literature that studies dynamic markets with adverse selection (e.g., Janssen and Roy (2002), Hörner and Vieille (2009), Fuchs and Skrzypacz (2012), Guerrieri and Shimer (2014), Fuchs et al. (2016), Daley and Green (2012, 2016)). Our innovation is the introduction of asset correlation, which allows us to study the information aggregation properties of these markets. This paper builds upon our previous work, Asriyan et al. (2017) (henceforth AFG), which demonstrates that multiple equilibria can exist in a model with two informed sellers. In this paper, we focus on the information aggregation properties of equilibria. In order to do so, we extend the two-seller model of AFG to a model with an arbitrary number of sellers. Characterizing the set of equilibria in the two-period model with an arbitrary number of sellers (Sections 2.4 2.5 follows closely from AFG. The main contribution of this paper is twofold. First, we explore the information aggregation properties of equilibria as the market grows large, and their implications for trade and welfare (Section 3). Second, we study the normative implications by considering the information design problem of a social planner who observes trading behavior and chooses what information to communicate to the

\footnotetext{
${ }^{3}$ See Bond et al. (2012) for a survey of both the theoretical and empirical literature on the real effects of information conveyed through markets.
} 
traders (Section 4). Here, we also contribute to the recently growing literature on information design (Bergemann and Morris, 2013, 2016). In addition to the information policy affecting the ex-ante incentives as in Boleslavsky and Kim (2018), we show how the endogeneity of the planner's information set constrains the optimal policy.

\section{The Model}

There are $N+1$ sellers indexed by $i \in\{1, \ldots, N+1\}$, with $N \geq 1$. Each seller is endowed with an indivisible asset and is privately informed of her asset's type, denoted by $\theta_{i} \in\{L, H\}$. Seller $i$ has a value $c_{\theta_{i}}$ for her asset, where $c_{L}<c_{H}$. The value of a type- $\theta$ asset to a buyer is $v_{\theta}$ and there is common knowledge of gains from trade, $v_{\theta}>c_{\theta}$.

We start by considering a model in which there are two trading periods: $t \in\{1,2\}$. We generalize our main results to an infinite-horizon model in the Appendix.4.$^{4}$ In each period, each seller is matched with two competing buyers who make private offers to the seller. Each buyer can make one offer; a buyer whose offer is rejected gets a payoff of zero and exits the game. The payoff to a buyer who purchases an asset of type $\theta$ at price $p$ is $v_{\theta}-p$.

Sellers discount future payoffs by a factor $\delta \in(0,1)$. The payoff to a seller with an asset of type $\theta$, who trades a price $p$ in period $t$ is

$$
\left(1-\delta^{t-1}\right) c_{\theta}+\delta^{t-1} p
$$

If the seller does not trade at either date, his payoff is $c_{\theta}$. One can interpret $c_{\theta}$ and $v_{\theta}$ as the present value of the flow payoffs from owning the asset to the seller and buyer respectively ${ }^{5}$ All players are risk neutral.

Asset values are correlated with an unobservable underlying state, $S$, that takes values in $\{\ell, h\}$. The unconditional distribution of $\theta_{i}$ is $\mathbb{P}\left(\theta_{i}=H\right)=\pi_{0} \in(0,1)$. Assets are mutually independent conditional on the state, but their conditional distributions are given by $\mathbb{P}\left(\theta_{i}=\right.$ $L \mid S=\ell)=\lambda \in\left(1-\pi_{0}, 1\right)$. To allow for arbitrarily high level of correlation, we set $\mathbb{P}(S=h)=$ $\pi_{0}$. Our correlation structure introduces the possibility that trades of one asset conveys relevant information about the aggregate state and therefore the value of other assets. To capture this possibility, we assume that all transactions are observable. Therefore, prior to making offers in the second period, each buyer observes the set of assets that traded in the first period.

Notice that by virtue of knowing her asset quality, each seller has a private and conditionally

\footnotetext{
${ }^{4}$ The two-period model facilitates a more precise characterization of the set of equilibria and thus a sharper intuition for our main results.

${ }^{5}$ Alternatively, we could specify the seller's payoff as $\delta^{t-1}\left(v_{\theta}-c_{\theta}\right)$ and interpret $c_{\theta}$ as the seller's production cost.
} 
independent signal about the aggregate state of nature. Thus, if each seller were to report her information truthfully to a central planner, the planner would learn the aggregate state with probability one as $N \rightarrow \infty$. Our interest is to explore under what conditions the same information can be gleaned from the transaction data of a decentralized market. To fix ideas, we assume that the aggregate state is publicly realized at the end of period 2 and ask whether the trading behavior in period 1 reveals the state $4^{6}$ To ensure that strategic interactions remain relevant, we focus on primitives which satisfy the following assumptions.

Assumption $1 \pi_{0} v_{H}+\left(1-\pi_{0}\right) v_{L}<c_{H}$.

Assumption $2 v_{L}<(1-\delta) c_{L}+\delta c_{H}$.

The first assumption, which we refer to as the "lemons" condition, asserts that the adverse selection problem is severe enough to rule out the efficient equilibrium in which all sellers trade immediately. In this equilibrium, trade is uninformative about the underlying state (regardless of $N)$. The second assumption implies a lower bound on the discount factor and ensures that dynamic considerations remain relevant. Our main results do not rely on this assumption but it simplifies exposition and rules out fully separating equilibria, which are also independent of $N$.

\subsection{Remarks on Modeling Assumptions}

To illustrate the key mechanism for our findings as clearly as possible, we have made several rather stark assumptions regarding the buyers' side of the market. In particular: buyers are short-lived in that they can only make one offer, they have identical values and information, and they compete in Bertrand fashion. This ensures that (1) a buyer makes zero (expected) profits on any accepted offer, and (2) there do not exist mutually agreeable unrealized gains from trades. The primary purpose of these assumptions is to isolate the reason by which trade is delayed (i.e., it is only due to the seller's strategic considerations) and to ensure that prices (i.e., buyers' offers) respond to new information.

While these features seem reasonable and are not strictly necessary for most our results, it is worth discussing them in a bit more detail. First, that buyers make offers in only one period is a fairly standard assumption in this literature (e.g., Swinkels, 1999, Kremer and Skrzypacz, 2007; Hörner and Vieille, 2009). The set of equilibrium outcomes we identify remain equilibrium outcomes in a model where buyers make offers over multiple periods provided those offers are publicly observable (though it is possible that other equilibria also exist). Complications

\footnotetext{
${ }^{6}$ In the Appendix, we demonstrate that our main results extend to an infinite horizon model in which the state is never (publicly) realized.
} 
arise when buyers are long-lived and offers are private as then a buyer may have incentive to experiment in the first period by making an offer that loses money if it is accepted in order to make profits conditional on a rejection (Deneckere and Liang, 2006; Daley and Green, 2018).

If buyers' values or information is not identical, then a seller may have incentive to delay trade in the first period in order to meet a more favorable buyer in the second period. The assumption that each buyer is matched to a single seller in a given period is purely for convenience and can easily be relaxed. In what follows, we will also assume that buyers in the second period observe first-period transactions but not prices. This too is simply for convenience. Because buyers are uninformed and make the offers using pure strategies in the first period, no additional information (beyond whether a transaction occured) is revealed by the price.

\subsection{Strategies and Equilibrium Concept}

A strategy of a buyer is a mapping from his information set to a probability distribution over offers. In the first period (i.e., at $t=1$ ), a buyer's information set is empty. In the second period, buyers' information set is a vector in $\{0,1\}^{N+1}$ which indicates whether each asset traded in the first period. If asset $i$ trades in the first period, then it is efficiently allocated and it is without loss to assume that buyers do not make offers for it in the second period (Milgrom and Stokey, 1982). The strategy of each seller is a mapping from her information set to a probability of acceptance. Seller $i$ 's information includes her type, the set of previous and current offers as well as the information set of buyers.

We use Perfect Bayesian Equilibria (PBE) as our solution concept. This has three im-

plications. First, each seller's acceptance rule must maximize her expected payoff at every information set taking buyers' strategies and the other sellers' acceptance rules as given (Seller Optimality). Second, any offer in the support of the buyer's strategy must maximize his expected payoff given his beliefs, other buyers' strategies and the seller's strategy (Buyer Optimality). Third, given their information set, buyers' beliefs are updated according to Bayes' rule whenever possible (Belief Consistency).

\section{$2.3 \quad$ Updating}

There are two ways by which a buyer's belief about seller $i$ is updated between the first and second period. First, they update their beliefs based on whether each asset traded. Let $\sigma_{i}^{\theta}$ denote the probability that buyers assign to seller $i$ trading in the first period if her asset is type $\theta$. Conditional on rejecting the offer in the first period, the buyers' interim belief is given 
by

$$
\pi_{i}^{I n t} \equiv \mathbb{P}\left(\theta_{i}=H \mid \text { reject at } t=1\right)=\frac{\pi_{0}\left(1-\sigma_{i}^{H}\right)}{\pi_{0}\left(1-\sigma_{i}^{H}\right)+\left(1-\pi_{0}\right)\left(1-\sigma_{i}^{L}\right)} .
$$

Second, before making offers in the second period, buyers learn about any other trades that took place in the first period. How this information is incorporated into the posterior depends on buyer beliefs about the trading strategy of the other sellers (i.e., $\sigma_{j}^{\theta}, j \neq i$ ). Let $z^{j} \in\{0,1\}$ denote the indicator for whether seller $j$ trades in the first period, and let $\mathbf{z}=\left(z^{j}\right)_{j=1}^{N+1}$ and $\mathbf{z}_{-i}=\left(z^{j}\right)_{j \neq i}$. Denote the probability of $\mathbf{z}_{-i}$ conditional on seller $i$ being of type $\theta$ by $\rho_{\theta}^{i}\left(\mathbf{z}_{-i}\right)$, which can be written as

$$
\rho_{\theta}^{i}\left(\mathbf{z}_{-i}\right) \equiv \sum_{s \in\{l, h\}} \mathbb{P}\left(S=s \mid \theta_{i}=\theta\right) \cdot \prod_{j \neq i} \mathbb{P}\left(z^{j} \mid S=s\right)
$$

where $\mathbb{P}\left(z^{j}=1 \mid S=s\right)=\sum_{\theta \in\{L, H\}} \sigma_{j}^{\theta} \cdot \mathbb{P}\left(\theta_{j}=\theta \mid S=s\right)$ is the probability that buyers assign to seller $j$ trading in state $s$. Provided there is positive probability that $i$ rejects the bid at $t=1$ and $\mathbf{z}_{-i}$ is realized, we can use equations (2) and (3) to express the posterior probability of seller $i$ being type $H$ conditional on these two events:

$$
\pi_{i}\left(\mathbf{z}_{-i}\right) \equiv \mathbb{P}\left(\theta_{i}=H \mid z^{i}=0, \mathbf{z}_{-i}\right)=\frac{\pi_{i}^{I n t} \cdot \rho_{H}^{i}\left(\mathbf{z}_{-i}\right)}{\pi_{i}^{I n t} \cdot \rho_{H}^{i}\left(\mathbf{z}_{-i}\right)+\left(1-\pi_{i}^{I n t}\right) \cdot \rho_{L}^{i}\left(\mathbf{z}_{-i}\right)}
$$

\subsection{Equilibrium Properties}

AFG establish several features that must hold in any equilibrium of the two-seller model. It is mostly straightforward to show that these properties extend to the model studied here with an arbitrary number of sellers. However, developing an intuition for them will be useful for understanding our main results in Sections 3 and 4 , so we provide some explanation of them here. Because they are generalizations of prior work, we will refer to them here as "properties" rather than lemmas or propositions.

In order to introduce them, we will use the following definitions and notation. We refer to the bid for asset $i$ at time $t$ as the maximal offer made across all buyers for asset $i$ at time $t$. Let $V(\tilde{\pi}) \equiv \tilde{\pi} v_{H}+(1-\tilde{\pi}) v_{L}$ denote buyers' expected value for an asset given an arbitrary belief $\tilde{\pi}$. Let $\bar{\pi} \in\left(\pi_{0}, 1\right)$ be such that $V(\bar{\pi})=c_{H}$, and recall that $\pi_{i}$ denotes the probability that buyers assign to $\theta_{i}=H$ prior to making offers in the second period.

Property 1 (Second period) If seller $i$ does not trade in the first period, then in the second period:

(i) If $\pi_{i}>\bar{\pi}$ then the bid is $V\left(\pi_{i}\right)$, which the seller accepts w.p.1. 
(ii) If $\pi_{i}<\bar{\pi}$ then the bid is $v_{L}$, which the high type rejects and the low type accepts w.p.1.

(iii) If $\pi_{i}=\bar{\pi}$, then with some probability $\phi_{i} \in[0,1]$, the bid is $c_{H}=V\left(\pi_{i}\right)$ and the seller accepts w.p.1. With probability $1-\phi_{i}$, the offer is $v_{L}$, the high type rejects, and the low type accepts w.p.1.

Note that a high type will only accept a bid higher than $c_{H}$. When the expected value of the asset is above $c_{H}$ (as in $(i)$ ), competition forces the equilibrium offer to be the expected value. When the expected value of the asset is below $c_{H}$ (as in $(i i)$ ), buyers cannot attract both types without making a loss. Thus, only the low type will trade and competition pushes the bid to $v_{L}$. Finally, when the expected value of the asset is exactly $c_{H}$ (as in $(i i i)$ ), buyers are indifferent between offering $c_{H}$ and trading with both types or offering $v_{L}$ and only trading with the low type.

Notice that Property 1 implies a second period payoff to a type- $\theta$ seller $i$ as a function of $\left(\pi_{i}, \phi_{i}\right)$, which we denote by $F_{\theta}\left(\pi_{i}, \phi_{i}\right)$, where

$$
F_{H}\left(\pi_{i}, \phi_{i}\right) \equiv \max \left\{c_{H}, V\left(\pi_{i}\right)\right\}
$$

and

$$
F_{L}\left(\pi_{i}, \phi_{i}\right) \equiv \begin{cases}v_{L} & \text { if } \pi_{i}<\bar{\pi} \\ \phi_{i} c_{H}+\left(1-\phi_{i}\right) v_{L} & \text { if } \pi_{i}=\bar{\pi} \\ V\left(\pi_{i}\right) & \text { if } \pi_{i}>\bar{\pi}\end{cases}
$$

Figure 1 plots the equilibrium price in the second period as a function of the posterior belief. Due to the sharp increase in the price at $\pi_{i}=\bar{\pi}$, the low type has a strong incentive to reject the first-period bid at beliefs just below $\bar{\pi}$ and wait for noisy information to be revealed - even if she expects the posterior to decrease. Thus, from seller $i$ 's perspective, the strategy of seller $j \neq i$ in the first period is relevant because it influences the distribution of news and therefore the distribution of $\pi_{i}$. In particular, the (expected) continuation value of a seller from rejecting an offer in the first period can be written as

$$
Q_{\theta}^{i} \equiv(1-\delta) c_{\theta}+\delta \sum_{\mathbf{z}_{-i}} \rho_{\theta}^{i}\left(\mathbf{z}_{-i}\right) F_{\theta}\left(\pi_{i}\left(\mathbf{z}_{-i}\right), \phi_{i}\right)
$$

It depends on seller $i$ 's own trading strategy $\sigma_{i}^{\theta}$ through the interim belief. But, importantly, it also depends on (i) the correlation of types with the state, and (ii) the strategies of other sellers, since both influence the distribution of "news" that the buyers receive about seller $i$ in the second period. 


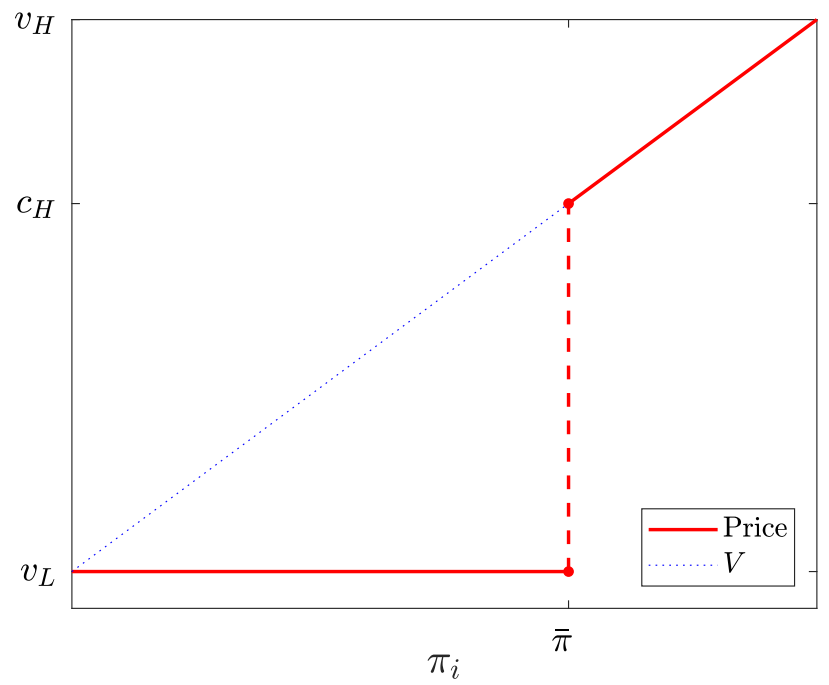

Figure 1: The equilibrium price in the second period as it depends on buyers' posterior belief.

Property 2 (Skimming) In any equilibrium, the expected continuation value of the high type is strictly greater than that for the low type: $Q_{H}^{i}>Q_{L}^{i}$.

This result, often referred to in the literature as a "skimming" property, is due to the fact that both the flow payoff $c_{\theta}$ and the continuation payoff $F_{\theta}$ are higher for the high type, and because the high type rationally expects a (weakly) better distribution of buyer posteriors (thus prices) in the second period.

Property 3 (First period) In the first period, the bid for each asset is $v_{L}$. The high-type seller rejects this bid with probability 1 . The low-type seller accepts it with probability $\sigma_{i}<1$.

By Property 2, any offer that is acceptable to a high type in the first period is accepted by the low type w.p.1. But Assumption 1 implies that any such offer yields negative profits for the buyers. Hence, in equilibrium only low types trade in the first period and competition pushes the bid to $v_{L}$. Finally, if $\sigma_{i}=1$, then the bid in the second period must be $v_{H}$ (Property 1). But then the low-type seller $i$ would strictly prefer to delay trade to the second period (Assumption 2), a contradiction.

Property 4 (Symmetry) In any equilibrium, $\sigma_{i}=\sigma>0$ for all $i$. If buyer mixing is part of the equilibrium then $\phi_{i}=\phi$ for all $i$.

The key step to prove symmetry is to show that if $\sigma_{i}>\sigma_{j} \geq 0$, then $Q_{L}^{i}>Q_{L}^{j}$. This follows from the fact that, due to imperfect correlation, $\pi_{i}$ (and therefore $Q_{L}^{i}$ ) is more sensitive to $i$ 's own trading probability than it is to that of the other sellers. Note that, by Property $3 . Q_{L}^{j} \geq v_{L}$. 
Hence, if $Q_{L}^{i}>Q_{L}^{j}$, then the low-type seller $i$ strictly prefers to wait, which contradicts $\sigma_{i}>0$ being consistent with an equilibrium. Next, note that it cannot be that $\sigma^{i}=0$ for all $i$. If that were the case, then no news arrives (since observing no trade contains no information) and buyers in the second period would have the same beliefs as buyers in the first period. This would imply that the second period bid is $v_{L}$ (Property 1), but in that case the low-type sellers would be strictly better off by accepting $v_{L}$ in the first period, which contradicts $\sigma_{i}=0$.

\subsection{Equilibria}

Given Properties 14, we will henceforth drop the $i$ subscripts wherever possible and denote a candidate equilibrium by the pair $(\sigma, \phi)$. Because all equilibria are symmetric, any information about seller $i$ that is contained in news $\mathbf{z}_{-i}$ does not depend on the identity of those who sold but only on the number (or fraction) of other sellers that traded. For example, suppose that $\mathbf{z}_{-i}=\mathbf{z}(K)$ where $\mathbf{z}(K)$ is such that $\sum_{j \neq i} z^{j}=K \leq N$. Then

$$
\rho_{\theta}^{i}(\mathbf{z}(K))=\sum_{s \in\{l, h\}} p_{s}^{K} \cdot\left(1-p_{s}\right)^{N-K} \cdot \mathbb{P}\left(S=s \mid \theta_{i}=\theta\right),
$$

where $p_{s} \equiv \sigma \cdot \mathbb{P}\left(\theta_{i}=L \mid S=s\right)$ is the probability that any given seller trades in state $s$. Naturally, the probability of observing $K$ trades among sellers $j \neq i$ is $\left(\begin{array}{l}N \\ K\end{array}\right) \cdot \rho_{\theta}^{i}(\mathbf{z}(K))$.

Furthermore, since any equilibrium involves $\sigma \in(0,1)$, a low-type seller must be indifferent between accepting $v_{L}$ in the first period and waiting until the second period. The set of equilibria can thus be characterized by the solutions to

$$
Q_{L}(\sigma, \phi)=v_{L},
$$

where we now make explicit the dependence of the continuation value on the strategy $(\sigma, \phi)$.

As we show in the next proposition, there can be multiple solutions to (8) and hence multiple equilibria.

Proposition 1 (Existence and Multiplicity) An equilibrium always exists. If $\lambda$ and $\delta$ are sufficiently large, there exist multiple equilibria.

Intuitively, a higher $\sigma$ has two opposing effects on the seller's continuation value. On the one hand, the posterior beliefs and thus prices in the second period are increasing in $\sigma$, which increases the expected continuation value $Q_{L}$. On the other hand, as other low types trade more aggressively, the distribution over buyers' posteriors shifts towards lower posteriors, thus decreasing $Q_{L}$. The latter force generates complementarities in sellers' trading strategies, which 
results in multiple equilibria when the correlation between assets is high and traders care sufficiently about the future.

We now turn to our main question, specifically, whether information about the underlying state is aggregated as the number of informed participants grows large. To understand the essence of this question, first notice that the trading behavior of each seller provides an informative signal about the aggregate state. If the seller trades in the first period, then she reveals her asset's type is $L$, which is more likely when the aggregate state is $\ell$ than when it is $h$. Conversely, if the seller does not trade, then buyers update their beliefs about the asset toward $H$ and their belief about the aggregate state toward $h$. Moreover, the amount of information revealed by each seller is increasing in the low-type's trading probability, which we now denote by $\sigma_{N}$ (in order to explicitly indicate its dependence on the number of other informed participants).

If the information content of each individual trade were to converge to some positive level (i.e., $\lim _{N \rightarrow \infty} \sigma_{N}=\bar{\sigma}>0$ ), then information about the state would aggregate. The reason is that by the law of large numbers the fraction of assets traded would concentrate around its population mean $\bar{\sigma} \cdot \mathbb{P}\left(\theta_{i}=L \mid S=s\right)$, which is strictly greater when the aggregate state is $\ell$ than when it is $h$. If, on the other hand, $\sigma_{N}$ decreases to zero at a rate weakly faster than $1 / N$ (i.e., $\lim _{N \rightarrow \infty} N \cdot \sigma_{N}<\infty$ ), then information would not aggregate. In this case, despite having arbitrarily many signals about the state, the informativeness of each signal goes to zero fast enough that the overall amount of information does not reveal the true state.

Of course, the equilibrium trading behavior of each individual seller is determined endogenously. Therefore, in order to establish information aggregation properties of equilibria, we need to understand how the set of equilibrium values of $\sigma_{N}$ changes with $N$. Moreover, since different equilibria have different $\sigma_{N}$, the limiting information aggregation properties could be different for different sequences of equilibria.

\section{Information Aggregation}

Consider a sequence of economies indexed by $N$ (standing for $N+1$ assets), and let $\sigma_{N}$ denote an equilibrium trading probability in the first period and $\pi_{N}^{\text {State }}$ be the buyers' posterior belief that the aggregate state is $h$, conditional on having observed the outcome of trade in the first period. That is, given a trading history $\mathbf{z}=\left(z^{j}\right)_{j=1}^{N+1}, \pi_{N}^{\text {State }}(\mathbf{z}) \equiv \mathbb{P}(S=h \mid \mathbf{z})$. We say that:

Definition 1 There is information aggregation along a given sequence of equilibria if $\pi_{N}^{\text {State }} \rightarrow^{p} 1_{\{S=h\}}$ as $N \rightarrow \infty$, where $\rightarrow^{p}$ denotes convergence in probability.

Our notion of information aggregation requires that, asymptotically, agents' beliefs about 
the aggregate state become degenerate at the truth. That our definition involves convergence in probability is standard in the literature (see e.g., Kremer (2002)).

Remark 1 In the two-period model, we focus on whether information aggregates based on trading in the first period (rather than the second period) because only information revealed in the first period has the potential to influence welfare. Any information revealed by second period trading is payoff irrelevant. We generalize our notion of information aggregation when analyzing the infinite horizon model in Appendix $D$.

\subsection{A 'Fictitious' Economy}

Before presenting our main results, it will be useful to consider a 'fictitious' economy in which buyers observe the true state, $S$, before making second period offers. This benchmark economy is useful because it approximates the information revealed in the true economy if there is information aggregation. We proceed by deriving a necessary and sufficient condition under which the fictitious economy supports an equilibrium with trade in the first period (Lemma 1). We then show that the same condition is necessary, though not sufficient, for information aggregation (Theorem 1). Intuitively, information aggregation requires trade. But if the fictitious economy does not support an equilibrium with trade, then (by continuity) there cannot exist a sequence of equilibria along which information aggregates.

First, note that Properties 1, 2, and 3 trivially extend to the fictitious economy. Second, observe that conditional on knowing the true state, when forming beliefs about seller $i$ the information revealed by sellers $j \neq i$ is irrelevant. That is, buyers' posterior belief about seller $i$ following a rejection in the first period and observing the true state is $s$ is given by

$$
\pi_{i}^{f i c t}(s)=\frac{\pi_{i}^{\text {Int }} \cdot \mathbb{P}\left(S=s \mid \theta_{i}=H\right)}{\pi_{i}^{\text {Int }} \cdot \mathbb{P}\left(S=s \mid \theta_{i}=H\right)+\left(1-\pi_{i}^{\text {Int }}\right) \cdot \mathbb{P}\left(S=s \mid \theta_{i}=L\right)},
$$

where $\pi_{i}^{I n t}$ is the interim belief given in (2). This implies that a seller's continuation value in the fictitious economy is independent of the trading strategies of the other sellers. Since there are no complementarities between sellers' trading strategies, the fictitious economy has a unique equilibrium, which must be symmetric (we will again drop the $i$ subscripts whenever possible). Analogous to (7), the continuation value is given by

$$
Q_{\theta}^{f i c t}(\sigma, \phi)=(1-\delta) c_{\theta}+\delta \sum_{s} \mathbb{P}(S=s \mid \theta) F_{\theta}\left(\pi^{f i c t}(s), \phi\right)
$$

As in Daley and Green (2012), due to the exogenous arrival of information, it is possible that the equilibrium of the fictitious economy involves zero probability of trade in the first period. 
Lemma 1 The unique equilibrium of the fictitious economy involves zero probability of trade in the first period (i.e., $\sigma^{\text {fict }}=0$ ) if and only if

$$
Q_{L}^{f i c t}(0,0) \geq v_{L}
$$

Furthermore, $(\bowtie)$ holds if and only if the parameters satisfy the following:

$$
\lambda \geq \bar{\lambda} \equiv 1-\frac{\pi_{0}(1-\bar{\pi})}{1-\pi_{0}}
$$

and

$$
\delta \geq \bar{\delta} \equiv \frac{v_{L}-c_{L}}{v_{L}-c_{L}+(1-\lambda) \cdot\left(1-\frac{(1-\lambda)\left(1-\pi_{0}\right)}{\pi_{0}}\right) \cdot\left(v_{H}-v_{L}\right)} .
$$

This result is intuitive. The equilibrium of the fictitious economy features no trade whenever the low type's option value from delaying trade to the second period is high. The first condition (i.e., $\lambda \geq \bar{\lambda}$ ) guarantees that, whenever $\sigma=0$, then the buyers' posteriors satisfy $\pi^{\text {fict }}(h)>\bar{\pi}$, which implies that the prospect of the state being revealed increases the expected prices (see Figure 1 and recall that $\pi_{0}<\bar{\pi}$ ). The second condition (i.e., $\delta \geq \bar{\delta}$ ) ensures that the cost of delay does not overwhelm the prospect of a higher price in the next period. Observe that $\bar{\delta}$ is increasing in $\lambda$ with $\lim _{\lambda \rightarrow 1} \bar{\delta}=1$.

\subsection{When Does Information Aggregate?}

We now establish our first main result, which shows that $(\bowtie)$ is also the crucial determinate of the information aggregation properties of equilibria.

\section{Theorem 1 (Aggregation Properties)}

(i) If $(\star)$ holds with strict inequality, then information aggregation fails along any sequence of equilibria.

(ii) If $₫$ does not hold, then there exists a sequence of equilibria along which information aggregates.

The proof of the first statement uses the observation that if information were to aggregate, then for $N$ large enough the continuation payoffs of the sellers are close to the continuation payoffs in the fictitious economy. Thus, when $(\star)$ holds strictly, delay is also uniquely optimal when there are a large but finite number of assets. But this contradicts Property 4 , which states that $\sigma_{N}=0$ cannot be part of an equilibrium for any finite $N$. In fact, when ( $\left.\star\right)$ holds strictly, 
$\sigma_{N}$ must go to zero at a rate proportional to $1 / N$, which is fast enough to prevent information from aggregating, but also slow enough to ensure that the transaction data does not become completely uninformative in the limit. If it did, the bid for any asset in the second period would be $v_{L}$ with probability arbitrarily close to one; hence low types would strictly prefer to trade in the first period (implying $\sigma_{N}=1$ ), which would also contradict Property 4 .

On the other hand, when the fictitious economy has an equilibrium with positive trade in the first period (i.e., if (太) does not hold), we can explicitly construct a sequence of equilibria in which the trading probability $\sigma_{N}$ is bounded away from zero. Clearly, information is aggregated along such a sequence. Nevertheless, even when aggregating equilibria exist, it is not the case that information will necessarily aggregate along every sequence of equilibria.

Theorem 2 (Coexistence) There exists a $\underline{\delta}<1$ such that whenever $\delta \in(\underline{\delta}, \bar{\delta})$ and $\lambda$ is sufficiently large, there coexists a sequence of equilibria along which information aggregates with a sequence of equilibria along which aggregation fails. If either $\lambda<\bar{\lambda}$ or $\delta$ is sufficiently small, then information aggregates along any sequence of equilibria.

To prove the first statement, we first note that for a given $\delta<1$, if $\lambda$ is sufficiently large, then we must have $\delta<\bar{\delta}$ ( since $\lim _{\lambda \rightarrow 1} \bar{\delta}=1$ ) and thus by Theorem 1 aggregating equilibria must exist. We then show that if we fix $\delta$ above a certain threshold, then for a sufficiently large $\lambda$, non-aggregating equilibria also exist. In particular, we explicitly construct a sequence of equilibria in which the second period bid is $v_{L}$ for all histories except the one in which no seller has traded in the first period. In these equilibria, the probability that no seller trades in the first period remains bounded away from zero, in both states of nature. Thus, even as $N \rightarrow \infty$, uncertainty about the state of nature does not vanish.

The second part of Theorem 2 provides sufficient conditions under which information necessarily aggregates. While this result is not particularly surprising, it is instructive to observe that the possibility of aggregation failure requires the two key ingredients of the model: $(1)$ sufficient correlation across assets (i.e. $\lambda>\bar{\lambda}$ ), and (2) that strategic delay is relevant (i.e. $\delta$ is large enough). Figure 2 illustrates and summarizes the findings in Theorems 1 and 2.

Remark 2 Our findings can be extended to a model with more than two trading periods. Intuitively, one might expect that with more trading periods there are more opportunities to learn from trading behavior and hence more information will be revealed. However, there is a countervailing force; there are more opportunities for (strategic) sellers to signal through delay. It turns out that two factors essentially cancel each other out. In Appendix $D$, we extend the model to allow for an infinite number of trading periods, generalize our definition of information aggregation, and demonstrate that the analogs of Theorems 1 and 2 continue to hold. 


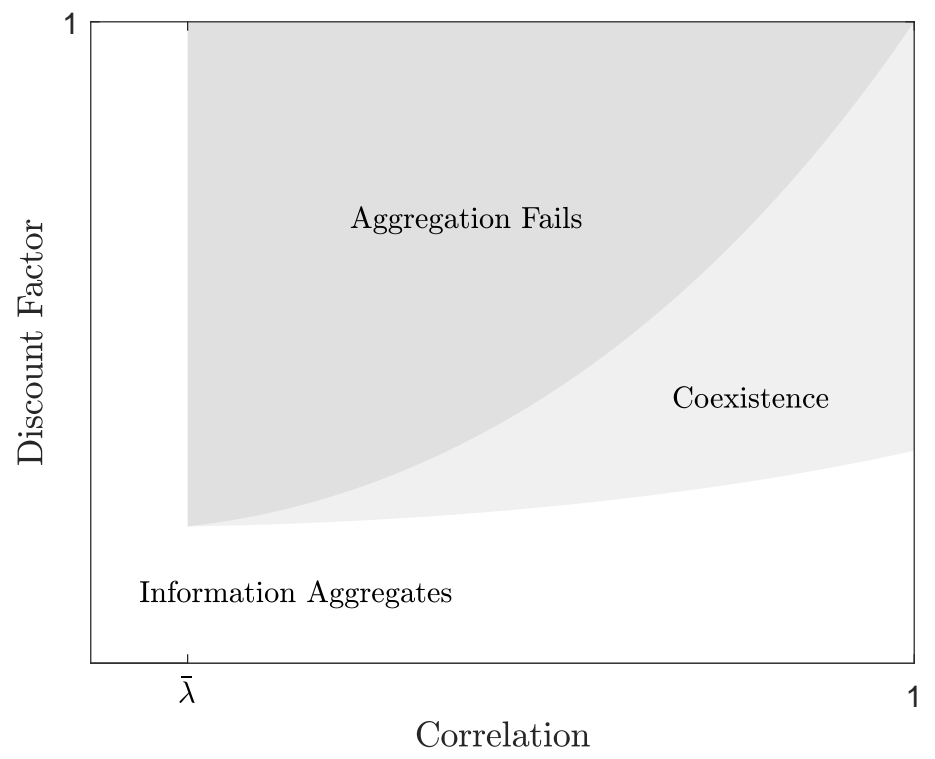

Figure 2: When does Information Aggregate? This figure illustrates the regions of the parameter space in which aggregating equilibria exist, fail to exist, or coexists with non-aggregating equilibria. In the top (darkly shaded) region, ( $\star$ ) holds and hence there do not exist sequences of equilibria that aggregate information. Otherwise, aggregating equilbria exist (Theorem 11). In the bottom-left (unshaded) region, all sequences of equilibria aggregate information and in the middle-right (lightly shaded) region, sequences in which information aggregates coexist with sequences in which information aggregation fails (Theorem 2 ).

\subsection{Trading Behavior and Welfare}

We now consider the implications of information aggregation for prices, trade volume, and welfare.

The ex-ante equilibrium surplus of a seller is:

$$
W_{N}=\left(1-\pi_{0}\right)\left(Q_{L, N}-c_{L}\right)+\pi_{0}\left(Q_{H, N}-c_{H}\right)
$$

where $Q_{\theta, N}$ is given by (7) when the market size is $N+1$. Because buyers are competitive and thus break even, $W_{N}$ is effectively the per trader surplus in our economy. As a benchmark for comparison, the per trader surplus in the unique equilibrium of the fictitious economy is:

$$
W^{f i c t}=\left(1-\pi_{0}\right)\left(Q_{L}^{f i c t}-c_{H}\right)+\pi_{0}\left(Q_{H}^{f i c t}-c_{H}\right),
$$

where $Q_{\theta}^{\text {fict }}$ is given by 10 . The following proposition shows that aggregating equilibria behave very much like the fictitious economy.

Proposition 2 (Aggregating Equilibria) Consider a sequence of equilibria along which information aggregates. Then, along this sequence: 

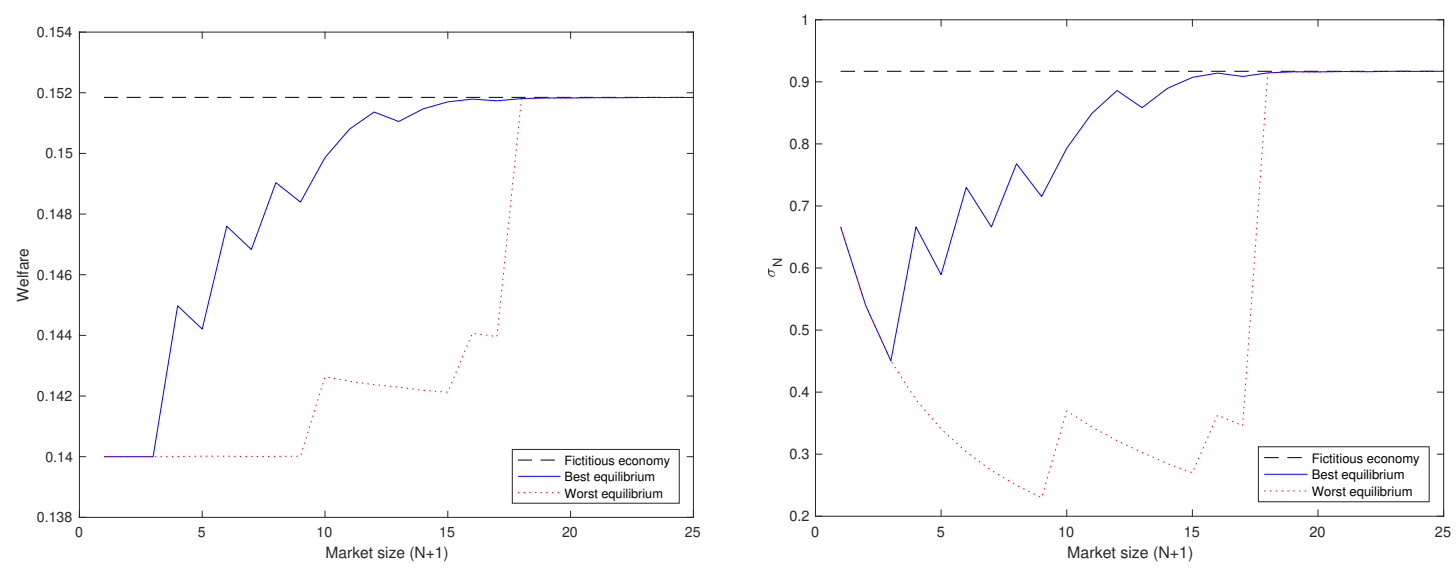

Figure 3: The left panel illustrates how the welfare per trader depends on the number of traders. The right panel shows the corresponding strategy of a low-type seller in the first period. The parameters are such that only aggregating equilibria exist.

(i) $\lim _{N \rightarrow \infty} W_{N}=W^{\text {fict }}$,

(ii) $\lim _{N \rightarrow \infty} \sigma_{N}=\sigma^{\text {fict }}$,

(iii) Conditional on the true state, the aggregate volatility of prices and of trading volume goes to zero.

Figure 3 illustrates this result graphically by plotting the equilibrium trading surplus $W_{N}$ and the trading probability $\sigma_{N}$ against the market size, $N+1$. For small $N$, multiple equilibria exist due to strategic complementarities among different sellers, and $W_{N}$ and $\sigma_{N}$ can either increase or decrease with $N$. As $N$ grows large, however, the aggregate state gets learned, the complementarities vanish, and both welfare and trading behaviour converge to those of the fictitious economy. The implication is that, in this economy, conditional on the aggregate state, the volatility in asset prices and trading volume (in both periods) goes to zero. As we show next, however, the picture changes dramatically in when information fails to aggregate.

Proposition 3 (Non-Aggregating Equilibria) Consider a sequence of equilibria such that information aggregation fails along any of its subsequences. Then, along this sequence:

(i) $\lim \sup _{N \rightarrow \infty} W_{N}<W^{\text {fict }}$,

(ii) $N \sigma_{N} \in(\underline{\kappa}, \bar{\kappa})$ for some constants $\underline{\kappa}, \bar{\kappa}>0$,

(iii) Conditional on the true state, the aggregate volatility of prices and of trading volume remains strictly positive. 

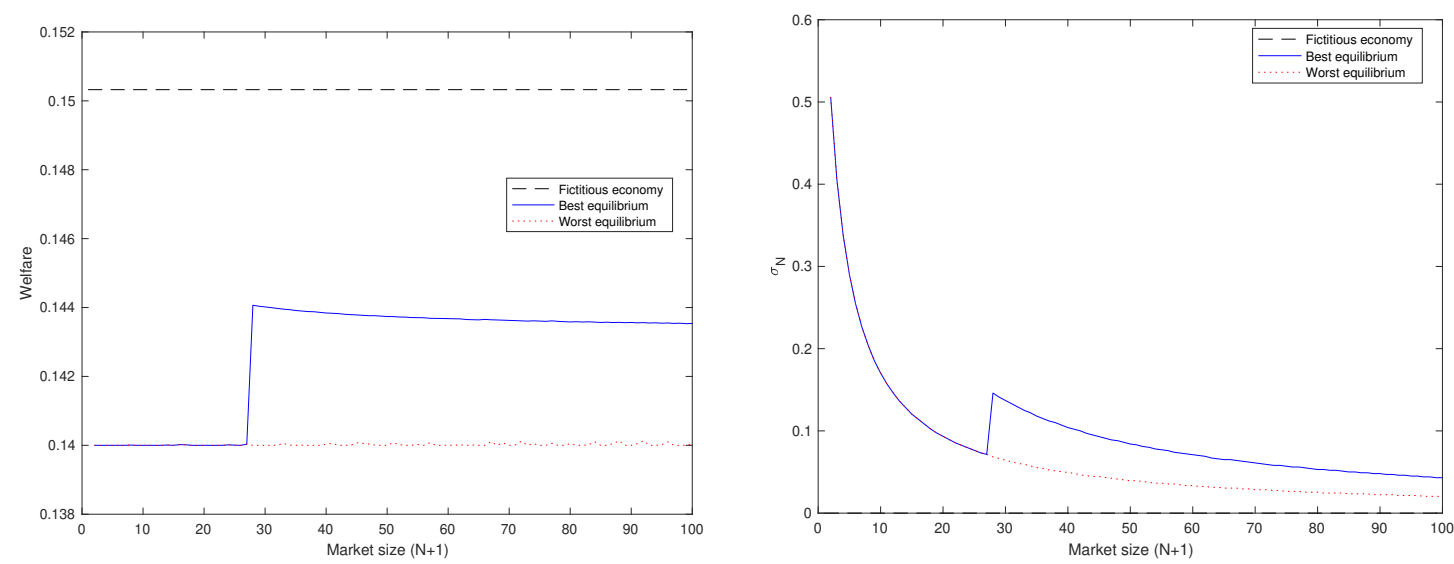

Figure 4: The left panel illustrates how the welfare per trader depends on the number of traders. The right panel shows the corresponding strategy of the seller in the first period. The parameters are such that $(\star)$ holds and, hence, aggregating equilibria do not exist.

In non-aggregating equilibria, strategic considerations do not vanish as the market grows large, which leads to (excess) volatility in prices conditional on the state and welfare that is below the fictitious benchmark. Figure 4 illustrates this result graphically.

The contrast between Propositions 2 and 3 demonstrates that aggregating equilibria have several nice properties that are not shared by their non-aggregating counterparts. Two immediate implications follow. First, from a social welfare perspective, aggregating equilibria are always preferable to non-aggregating equilibria when they co-exist. Thus, among laissez-faire outcomes, aggregation is optimal. Second, if the only laissez-faire outcomes are non-aggregating and a social planner could manage to learn the true state in the first period, then she could improve welfare by revealing her information to market participants.

Of course, it is not obvious how a planner would be able to acquire such information. It is more natural to think that the planner is ex-ante uninformed, but can learn about the true state by observing the trading behavior of market participants. The problem facing the planner is then how best to reveal this information to other agents in the economy. In the next section, we tackle precisely this problem.$^{7}$

\footnotetext{
${ }^{7}$ An alternative way to facilitate aggregation is through speculation; by introducing an Arrow Debreu security that is traded on a centralized market at $t=1$, whose payoff depends on the true state, which (by assumption) is publicly realized at $t=2$. As $N \rightarrow \infty$, the market clearing price for the security will reveal the true state and welfare will converge to that in the fictitious economy. Speculation weakly improves welfare compared to the laissez-faire outcome. However, as we will see in Section 4, introducing speculation does not necessarily lead to the constrained efficient outcome.
} 


\section{Optimal Information Policy}

How should an (uninformed) planner disclose trading behavior to maximize social welfare? Before analyzing the planner's problem, it is useful to compare the problem we consider to the literature on Bayesian persuasion (Kamenica and Gentzkow, 2011; Rayo and Segal, 2010) and "information design" problems more generally (Bergemann and Morris, 2013, 2016) 8 On one hand, the problems are quite similar. Both involve designing an information revelation policy to induce other players to take certain actions. On the other hand, the planner's problem in our setting must take into account a novel feedback effect. Namely, the planner's policy influences the information content of trading behavior, and therefore the information content of whatever is revealed. In short, the statistical properties of the information the planner can reveal, which is typically exogenous in a Bayesian persuasion setting, depend on the policy itself.

Our solution method for answering this question will proceed in two steps. First, we consider the information design problem of a planner who (exogenously) learns the aggregate state in the first period. We refer to this as the Informed Planner's Problem. We characterize the solution to this problem, which for $\delta$ large enough involves partially concealing the aggregate state in order to increase the trading surplus compared to the laissez-faire outcome. We then return to the problem of interest and provide the necessary and sufficient conditions under which the (uninformed) planner can achieve the same welfare as the informed planner. When these conditions do not hold, the welfare is strictly lower than when the planner is informed. Finally, we relate our normative findings to the information aggregation properties of equilibria.

\subsection{Informed Planner's Problem}

In this section, we set up the informed planner's problem and characterize its solution. In doing so, we will assume that the planner (exogenously) learns the aggregate state at $t=1$ and can design and commit to an information policy ex-ante, the results of which are publicly revealed after trading at $t=1$. Therefore, buyers of asset $i$ at date $t=2$ can observe (i) whether asset $i$ traded in the first period, and (ii) any additional information revealed by the planner.

The planner's objective is to maximize the expected discounted gains from trade. Because we focus on a public information policy and all assets are ex-ante identical, it is sufficient to consider the problem of maximizing the expected discounted gains from trade for a single asset.

\footnotetext{
8 Bergemann and Morris (2017) provide a more general treatment of information design problems drawing a distinction between whether the designer has an informational advantage (as in Bayesian persuasion) or not (as in communication games). In our model, the planner has no informational advantage ex-ante but has a technology for acquiring one in the interim. Another important distinction of our setting is that the planner has only limited means by which she can elicit information.
} 
The planner's objective can be written as

$$
W=\left(1-\pi_{0}\right)(\sigma+\delta(1-\sigma))\left(v_{L}-c_{L}\right)+\pi_{0}(\mathbb{P}(\pi=\bar{\pi} \mid H) \phi+\mathbb{P}(\pi>\bar{\pi} \mid H)) \delta\left(v_{H}-c_{H}\right)
$$

where $\pi$ is the (random) buyers' posterior belief at $t=2$ that the seller is a high type.

From Kamenica and Gentzkow (2011), the problem of choosing state-dependent distributions over signals is equivalent to choosing a distribution of posteriors about the state that is Bayes plausible. Let $\tilde{p}$ denote the random variable representing the buyers' posterior about the state conditional on observing the information revealed by the planner and let $G$ denote the cumulative distribution of $\tilde{p}$. Bayes plausbility requires that the expected posterior be equal to the prior

$$
E_{G}\{\tilde{p}\}=\pi_{0} .
$$

Of course, the planner's choice of $G$ will influence both the behavior of the seller and the buyers as captured by $(\sigma, \phi)$. We refer to $G$ as the information policy of the informed planner.

Definition 2 (Informed Planner's Problem) The informed planner's problem is to choose a triple $(G, \sigma, \phi)$ to maximize (11) subject to two constraints:

(1) Bayes Plausibility (i.e., (12)), and

(2) Given $G,(\sigma, \phi)$ must be an equilibrium of the game.

We say that $(G, \sigma, \phi)$ is feasible if it satisfies (1) and (2). We let $Q_{\theta}^{G}(\sigma, \phi)$ denote the continuation value to a type- $\theta$ seller 9 F For any information policy, Properties 1,3 must hold in any equilibrium. Moreover, as in Section 2.5, if $\sigma \in(0,1)$ then constraint $(2)$ requires that the low type's continuation value must equal $v_{L}$. However, with an informed planner, it is no longer true that low type sellers must trade with strictly positive probability in the first period (as in Property 4). More specifically, it is possible to design $G$ such that there exist equilibria in which $\sigma=0$ and $Q_{L}^{G} \geq v_{L}$. Instead of (8), equilibria are characterized by $(G, \sigma, \phi)$ such that

$$
\begin{aligned}
Q_{L}^{G}(\sigma, \phi) & \geq v_{L} \\
\sigma\left(Q_{L}^{G}(\sigma, \phi)-v_{L}\right) & =0
\end{aligned}
$$

It is convenient to let $\pi(\tilde{p}, \sigma)$ denote the buyers' posterior about the seller following a rejection in the first period, conditional on the buyers' posterior about the state being $\tilde{p}$. The following lemma puts a bound on the set of feasible $\sigma$ that can be implemented.

\footnotetext{
${ }^{9}$ See Appendix $\mathrm{C}$ for an explicit construction of the continuation values.
} 
Lemma 2 Define $\bar{\sigma}$ implicitly by $\pi(0, \bar{\sigma})=\bar{\pi}$ and define $\underline{\sigma} \equiv \inf \{\sigma \in[0,1], \pi(1, \sigma) \geq \bar{\pi}\}$, then $\sigma$ is feasible only if $\sigma \in[\underline{\sigma}, \bar{\sigma}]$.

The proof is simple. If $\sigma>\bar{\sigma}(<\underline{\sigma})$, then $Q_{L}^{G}>v_{L}\left(<v_{L}\right)$ regardless of what information is revealed by the planner. The next lemma simplifies the informed planner's problem by showing that, for any candidate $\sigma$, it is enough to consider information policies with at most three beliefs in the support.

Lemma 3 The solution to the informed planner's problem can be achieved with an information policy that has support $\Sigma(\sigma) \subseteq\{0, \bar{p}(\sigma), 1\}$, for some feasible $\sigma$ and $\bar{p}(\sigma)$ s.t. $\pi(\bar{p}(\sigma), \sigma)=\bar{\pi}$.

The intuition behind Lemma 3 is as follows. Take any $(G, \sigma, \phi)$ such that $\hat{p} \in(0, \bar{p}(\sigma))$ is in the support of $G$. The low type's payoff in the second period following the realization of $\hat{p}$ is $v_{L}$ and the high type gets $c_{H}$. The same payoffs can be achieved by a policy that reveals either $\tilde{p}=0$ or $\tilde{p}=\bar{p}(\sigma)$ and where $\phi$ is adjusted down to keep the low type indifferent. Thus, it is without loss to restrict attention to policies that do not involve posteriors $\tilde{p} \in(0, \bar{p}(\sigma))$.

Next, consider any policy $(G, \sigma, \phi)$ such that $\hat{p} \in(\bar{p}(\sigma), 1)$ is in the support of $G$. Let $G^{\prime}$ be a new information policy that reassigns the weight on $\hat{p}$ to $\bar{p}(\sigma)$ and 1 (respecting Bayesian plausibility). It can be shown that $Q_{H}^{G^{\prime}}(\sigma, \phi) \geq Q_{H}^{G}(\sigma, \phi)$ and $Q_{L}^{G^{\prime}}(\sigma, \phi) \leq Q_{L}^{G}(\sigma, \phi)$. Therefore, it is possible to find $\sigma^{\prime} \geq \sigma$ such that $\left(G^{\prime}, \sigma^{\prime}, \phi\right)$ is a feasible policy under which both seller types are weakly better off.

Thus, for any given $\sigma$, the information policy (i.e., $G$ ) of the informed planner has been reduced to choosing a pair $\left(\mu_{0}, \mu_{1}\right) \in[0,1]^{2}$, where $\mu_{k}=\mathbb{P}^{G}(\tilde{p}=k)$, and the Bayes plausibility constraint reduces to

$$
\mu_{1}+\left(1-\mu_{0}-\mu_{1}\right) \bar{p}(\sigma)=\pi_{0}
$$

Definition 3 We say that the planner's policy is fully revealing if $\mu_{0}=1-\pi_{0}$ and $\mu_{1}=\pi_{0}$.

If the policy attaches a strictly positive weight to $\bar{p}(\sigma)$ (i.e., if $\mu_{0}+\mu_{1}<1$ ) then some information is concealed. To further characterize the solution, it is useful to first consider a modified version of the problem, in which 13 is required to hold with equality.

When constraint (13) holds with equality, the planner's objective reduces to maximizing the payoff of the high-type seller, as given by

$$
Q_{H}^{G}=c_{H}+\delta \cdot \frac{\mathbb{P}(S=h \mid \theta=H)}{\pi_{0}} \cdot \mu_{1} \cdot\left(V(\pi(1 ; \sigma))-c_{H}\right) .
$$

Note that $Q_{H}^{G}$ is increasing in $\mu_{1}$ since the planner reveals that the state is $h$ more frequently (and the price in that event is highest), and it is increasing in $\sigma$ since the pooling price in that 
state is higher. Crucially, whether the planner faces a tradeoff between $\mu_{1}$ and $\sigma$ depends on whether revealing the state more frequently increases the low type's payoff from delay.

Lemma 4 Consider a variant of the informed planner's problem in which (13) is required to hold with equality. Recalling that $Q_{L}^{\text {fict }}(\sigma, \phi)$ is defined in 10$)$, the solution to this modified problem is as follows:

(i) If $Q_{L}^{\text {fict }}(\bar{\sigma}, 0) \leq v_{L}$, then the optimal information policy is fully revealing, $\sigma^{*}=\bar{\sigma}$, and $\phi^{*}$ is such that $Q_{L}^{\text {fict }}\left(\bar{\sigma}, \phi^{*}\right)=v_{L}$.

(ii) If $Q_{L}^{f i c t}(\bar{\sigma}, 0)>v_{L}$, then the optimal information policy conceals some information: $\mu_{0}^{*}=$ $0, \mu_{1}^{*}=\frac{\pi_{0}-\bar{p}\left(\sigma^{*}\right)}{1-\bar{p}\left(\sigma^{*}\right)}$, and $\sigma^{*}$ is such that $Q_{L}^{G^{*}}\left(\sigma^{*}, 0\right)=v_{L}$,

Intuitively, the reason why the planner conceals information is closely related to the option value effect of information that we identified in the fictitious economy of Section 3.1; the prospect of the high state being revealed more frequently can generate an increase in the low type's expected future prices and thus reduces her incentive to trade in the first period.

Lemma 4 will be useful in the study of the uninformed planner's problem. Before moving to that problem, however, we complete the characterization of the informed planner's true problem.

Proposition 4 The solution to the informed planner's problem is as follows.

(i) The constraint (13) is slack and the optimal policy involves $\sigma^{*}=\mu_{1}^{*}=0$ if and only if $\delta$ is sufficiently close to 1.

(ii) Otherwise, the constraint (13) holds with equality and the optimal policy is characterized by Lemma 4 .

If 13 is slack as in part (i) of Proposition 4, then it must be that the low-type seller trades with probability zero in the first period (see (14)). In this case, the planner's problem reduces to maximizing the probability of trade with the high type in the second period. Proposition 4 says that this is accomplished by never fully revealing the high state. As we will see in the next section, such a policy is not feasible for the uninformed planner.

\subsection{Uninformed Planner's Problem}

We are now ready to tackle our problem of interest where, rather than being endowed exogenously with knowledge of the state, the planner must learn it from transaction data. As a result, we will need to keep track of the market size, $N+1$, since it will affect the information 
the planner observes. That the planner's information is endogenously determined also makes it cumbersome to employ the typical Bayesian persuasion approach (i.e., choose a distribution over posterior beliefs subject to Bayes plausibility) that has become standard in the literature and which we adopted with the informed planner 10 Instead, we will work directly with the planner's reporting policy, which is defined as a mapping $\mathcal{M}_{N}$ from the trading histories she observes, which are elements of $\{0,1\}^{N+1}$, to distributions over signals that are publicly observed by agents in the economy. As before, the planner's objective is to maximize the expected discounted gains from trade,

$W^{\mathcal{M}_{N}}=\left(1-\pi_{0}\right)\left(\sigma_{N}+\delta\left(1-\sigma_{N}\right)\right)\left(v_{L}-c_{L}\right)+\pi_{0}\left(\mathbb{P}^{\mathcal{M}_{N}}(\pi=\bar{\pi} \mid H) \phi_{N}+\mathbb{P}^{\mathcal{M}_{N}}(\pi>\bar{\pi} \mid H)\right) \delta\left(v_{H}-c_{H}\right)$,

where $\mathbb{P}^{\mathcal{M}_{N}}(\cdot \mid \theta)$ denotes the conditional probability distribution over the buyers' posteriors induced by $\mathcal{M}_{N}$. We let $Q_{\theta}^{\mathcal{M}_{N}}\left(\sigma_{N}, \phi_{N}\right)$ denote the continuation value to a type- $\theta$ seller.

Definition 4 (Uninformed Planner's Problem) The uninformed planner's problem is to choose a triple $\left(\mathcal{M}_{N}, \sigma_{N}, \phi_{N}\right)$ to maximize (16) subject to $\left(\sigma_{N}, \phi_{N}\right)$ being an equilibrium of the game given the reporting policy $\mathcal{M}_{N}$.

The difference from the informed planner's problem is that the information content of any signal revealed by the planner is endogenous to the equilibrium trading probability $\sigma_{N}$. This has the following important implication.

Lemma 5 The solution to the uninformed planner's problem must involve $\sigma_{N}>0$ and

$$
Q_{L}^{\mathcal{M}_{N}}\left(\sigma_{N}, \phi_{N}\right)=v_{L}
$$

Intuitively, if $\sigma_{N}$ is equal to zero, the planner has no relevant information and any signals she reveals are completely uninformative. But, by the same argument used to establish Property 4. "no trade" cannot be part of an equilibrium if no information is revealed at $t=1$.

Lemma 5 implies that the solution to the modified informed planner's problem characterized in Lemma 4 provides an upper bound on the level of surplus that the uninformed planner can achieve. Clearly, a policy for the uninformed planner that achieves this upper bound must also be optimal for any $\delta$.

Proposition 5 There exists a sequence $\left\{\mathcal{M}_{N}, \sigma_{N}, \phi_{N}\right\}$ such that $\lim _{N \rightarrow \infty} W^{\mathcal{M}_{N}}=W^{*}$, where $W^{*}$ is the trading surplus under the information policy of the modified informed planner's problem as characterized in Lemma 4 .

\footnotetext{
${ }^{10}$ To employ the Bayesian persuasion approach for the uninformed planner's problem, Bayesian plausibility would need to be supplemented with an additional constraint on the support of the distribution (i.e., the planner cannot reveal information that she does not have), where the additional constraint depends on both $N$ and $\sigma_{N}$.
} 
To prove this result, we construct an information policy consisting of a binary signal, $\omega_{N} \in$ $\{b, g\}$, where the planner sends signal $\omega_{N}=g$ with probability $\mu_{1}^{*}$ when she observes that the fraction of sellers who traded at $t=1$ is below some threshold $\tau \in(0,1)$, and she sends signal $\omega_{N}=b$ otherwise. We show that, when $\sigma_{N}$ is close to $\sigma^{*}$ and $\tau$ is chosen appropriately, the planner asymptotically learns the state and the information content of her policy converges to that of the information policy of the informed planner as characterized in Lemma 4, which may or may not be fully revealing. Finally, we use continuity arguments to find a sequence of trading probabilities $\sigma_{N}$ which both converges to $\sigma^{*}$ and is consistent with equilibrium under this information policy, for all $N$.

The optimal information policy achieves a Pareto improvement over the laissez-faire outcomes. The reason is that the buyers break even, the low type's payoff is $v_{L}$ (see Lemma 5 ) and, thus, all the additional surplus generated by the planner's policy is captured by the high types. The implication of this observation is that all agents would be happy to delegate the information dissemination about past trades to the social planner.

Finally, taking Proposition 4 and Lemma 5 together, we can see that for $\delta$ large enough, the uninformed planner cannot achieve the same level of surplus as the informed planner, even as $N \rightarrow \infty$.

\subsection{Is Information Aggregation Constrained Efficient?}

Having characterized the solution to the uninformed planner's problem, we now relate our findings on optimal policy to the results on Information Aggregation in Section 3 . In order to do so, we will refer to any equilibrium that coincides with the solution to the uninformed planner's problem as constrained efficient, and we will refer to any equilibrium in which welfare is strictly below the solution to the uninformed planner's problem as constrained inefficient.

Corollary 1 Non-aggregating equilibria are constrained inefficient.

Not surprisingly, there is always scope for intervention if the laissez-faire outcome does not aggregate information. The reason for this is that, since the equilibrium is non-aggregating, there is a vanishing amount of trade in the first period, which is inefficient. Moreover, because the equilibrium is non-aggregating, there is little information revealed in the second period, and thus the adverse selection problem remains severe. However, even when an aggregating equilibrium exists and agents coordinate on playing it, there may also be scope for intervention.

Corollary 2 Aggregating equilibria are constrained inefficient if and only if

$$
Q_{L}^{f i c t}(\bar{\sigma}, 0) \geq v_{L}
$$




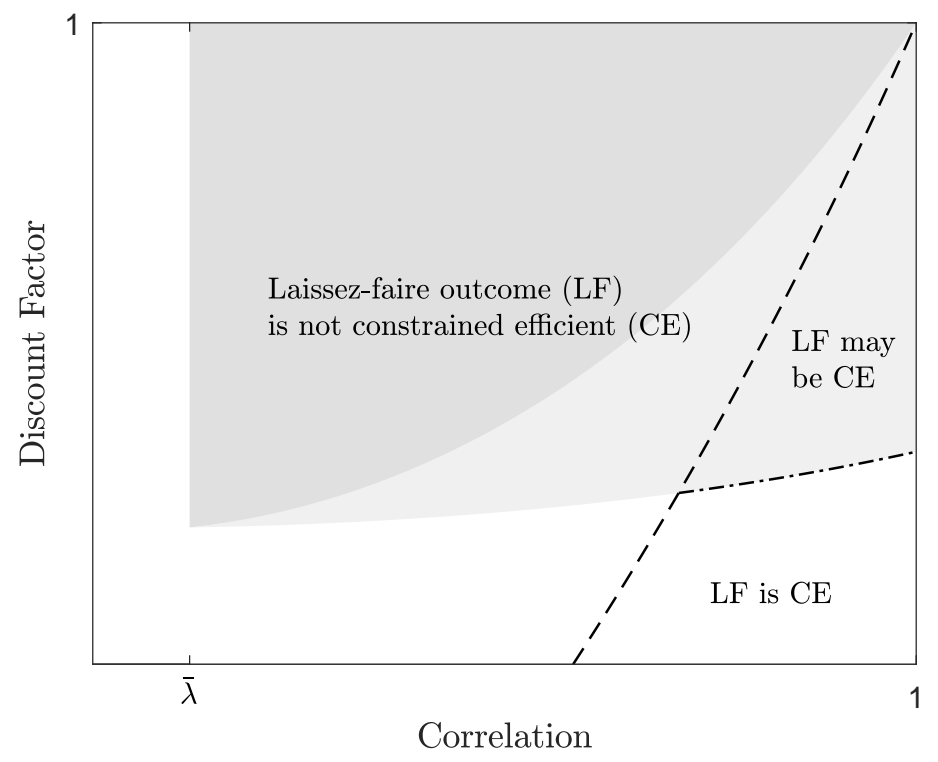

Figure 5: When is the Laissez-Faire Outcome Constrained Efficient? This figure relates the constrained efficiency of the laissez-faire outcome to whether information aggregates. The shaded regions are analogous to those in Figure 2 (i.e., aggregation fails in the darkest shaded region, there is coexistence in the lighter shaded region, and information necessarily aggregates in the unshaded region). The black dashed line is $\widehat{\delta}$. By Corollary 2. aggregating equilibria are constrained efficient only to the right of this line.

which holds if and only if the parameters satisfy the following:

$$
\delta \geq \widehat{\delta} \equiv \frac{v_{L}-c_{L}}{v_{L}-c_{L}+(1-\lambda) \pi(1 ; \bar{\sigma})\left(v_{H}-v_{L}\right)}
$$

It is worth noting that $\widehat{\delta}$ lies strictly below $\bar{\delta}$ defined in Lemma 1 , since $Q_{L}^{\text {fict }}(\bar{\sigma}, 0)>$ $Q_{L}^{f i c t}(0,0)$. Furthermore, $\widehat{\delta}$ is increasing in $\lambda$ with $\lim _{\lambda \rightarrow 1} \widehat{\delta}=1$. Therefore, the region where aggregating and non-aggregating equilibria coexist is bisected by $\widehat{\delta}$ as illustrated in Figure 5 .

\section{Concluding Remarks}

We study the information aggregation properties of decentralized dynamic markets in which traders have private information about the value of their asset, which is correlated with some underlying 'aggregate' state of nature. We provide necessary and sufficient conditions under which information aggregation necessarily fails. Further, we show that when these conditions are violated, there can be a coexistence of non-trivial equilibria in which information about the state aggregates with equilibria in which aggregation fails. Our findings suggest there are important differences in the aggregation properties of multi-asset decentralized markets (as studied here) and single-asset centralized markets as typically explored in the literature. 
We then consider the normative implications of our theory. We solve for the optimal information policy of a social planner who observes the trading behavior and chooses what information to communicate to the traders. We show that there is a relationship between information aggregation properties of equilibria and their efficiency. When information fails to aggregate, there is room for a planner to improve the outcome. In some cases, the outcome can also be improved upon when information does aggregate. Generally speaking, the planner achieves higher welfare with an information policy that is not fully revealing. This policy effectively conceals favorable "news" from the agents in order to accelerate trade, suggesting that full transparency may not always be optimal from a social welfare perspective. 


\section{References}

Albagli, E., A. Tsyvinski, And C. Hellwig (2015): "A Theory of Asset Prices Based on Heterogeneous Information," Working Paper.

Asriyan, V., W. Fuchs, And B. Green (2017): "Information Spillovers in Asset Markets with Correlated Values," American Economic Review, 107(7), 2007-40.

Axelson, U. And I. Makarov (2017): "Informational Black Holes in Financial Markets," Working Paper.

Babus, A. And P. Kondor (2016): "Trading and Information Diffusion in Over-the-Counter Markets," Working Paper.

Back, K., C. H. CaO, and G. A. Willard (2000): "Imperfect Competition Among Informed Traders," Journal of Finance, 55(5), 2117-2155.

Baumol, W. J. (1965): The Stock Market and Economic Efficiency, New York: Fordham University Press.

Bergemann, D. And S. Morris (2013): "Robust Predictions in Games with Incomplete Information," Econometrica, 81, 1251-1308.

(2016): "Bayes Correlated Equilibrium and the Comparison of Information Structures in Games," Theoretical Economics, 11, 487-522.

(2017): "Information Design: A Unified Perspective," Working Paper.

Bernanke, B. S. And M. WoOdford (1997): "Inflation forecasts and monetary policy," Tech. rep., National Bureau of Economic Research.

Bodoh-Creed, A. (2013): "Efficiency and Information Aggregation in Large Uniform-Price Auctions," Journal of Economic Theory, 146, 2436-2466.

Boleslavsky, R., D. L. Kelly, and C. R. Taylor (2017): "Selloffs, bailouts, and feedback: Can asset markets inform policy?" Journal of Economic Theory, 169, 294-343.

Boleslavsky, R. And K. Kim (2018): "Bayesian persuasion and moral hazard," .

Bond, P., A. Edmans, And I. Goldstein (2012): "The Real Effects of Financial Markets," Annual Review of Financial Economics, 4, 339-360.

Bond, P. And I. Goldstein (2015): "Government intervention and information aggregation by prices," The Journal of Finance, 70, 2777-2812.

Bond, P., I. Goldstein, And E. S. Prescott (2009): "Market-based corrective actions," The Review of Financial Studies, 23, 781-820.

Camargo, B., K. Kim, And B. Lester (2015): "Information spillovers, gains from trade, 
and interventions in frozen markets," The Review of Financial Studies, 29, 1291-1329.

Daley, B. And B. Green (2012): "Waiting for News in the Market for Lemons," Econometrica, 80(4), 1433-1504.

(2016): "An Information-Based Theory of Time-Varying Liquidity," Journal of Finance, $71(2), 809-870$.

Daley, B. And B. S. Green (2018): "Bargaining and News," Working Paper.

Deneckere, R. And M.-Y. Liang (2006): "Bargaining with Interdependent Values," Econometrica, 74, 1309-1364.

Dow, J. AND G. GorTon (1997): "Stock market efficiency and economic efficiency: Is there a connection?" The Journal of Finance, 52, 1087-1129.

Duffie, D., P. Dworczak, And H. Zhu (2017): "Benchmarks in Search Markets," Journal of Finance (forthcoming).

Fishman, M. J. And K. M. Hagerty (1989): "Disclosure decisions by firms and the competition for price efficiency," The Journal of Finance, 44, 633-646.

- (1992): "Insider trading and the efficiency of stock prices," The RAND Journal of Economics, 106-122.

Foster, F. D. And S. Viswanathan (1996): "Strategic Trading when Agents Forecast the Forecasts of Others," Journal of Finance, 51(4), 1437-1478.

Fuchs, W., A. ÖEry, AND A. Skrzypacz (2016): "Transparency and Distressed Sales under Asymmetric Information," Theoretical Economics, 11, 1103-1144.

Fuchs, W. And A. A. Skrzypacz (2012): "Costs and benefits of dynamic trading in a lemons market," Working Paper, 1-33.

Golosov, M., G. Lorenzoni, And A. Tsyvinski (2014): "Decentralized trading with private information," Econometrica, 82, 1055-1091.

Grossman, S. (1976): "On the efficiency of competitive stock markets where trades have diverse information," The Journal of Finance, 31, 573-585.

Guerrieri, V. AND R. Shimer (2014): "Dynamic adverse selection: A theory of illiquidity, fire sales, and flight to quality," American Economic Review, 104, 1875-1908.

Hayek, F. A. (1945): "The Use of Knowledge in Society," American Economic Review, 35, 519-530.

HellwiG, M. F. (1980): "On the aggregation of information in competitive markets," Journal of economic theory, 22, 477-498. 
Hörner, J. And N. Vieille (2009): "Public vs. Private Offers in the Market for Lemons," Econometrica, 77(1), 29-69.

Janssen, M. C. W. And S. Roy (2002): "Dynamic Trading in a Durable Good Market with Asymmetric Information," International Economic Review, 43:1, 257-282.

Kamenica, E. And M. Gentzkow (2011): "Bayesian persuasion," The American Economic Review, 101, 2590-2615.

Kremer, I. (2002): "Information Aggregation in Common Value Auctions," Econometrica, $70,1675-1682$.

Kremer, I. And A. Skrzypacz (2007): "Dynamic Signaling and Market Breakdown," Journal of Economic Theory, 133, 58-82.

Kyle, A. S. (1985): "Continuous auctions and insider trading," Econometrica, 53(6), 13151335.

(1989): "Informed speculation with imperfect competition," The Review of Economic Studies, 56, 317-355.

Lauermann, S. And A. Wolinsky (2016): "Search with Adverse Selection," Econometrica, 84(1), 243-315.

- (2017): "Bidder Solicitation, Adverse Selection, and the Failure of Competition," American Economic Review, 107, 1399-1429.

Leland, H. E. (1992): "Insider trading: Should it be prohibited?" Journal of Political Economy, 100, 859-887.

Lester, B., A. Shourideh, V. Venkateswaran, and A. Zetlin-Jones (2018): "Marketmaking with Search and Information Frictions," Tech. rep., National Bureau of Economic Research.

Maestri, L., D. Gerardi, B. Camargo, et al. (2016): "Efficiency in Decentralized Markets with Aggregate Uncertainty," in 2016 Meeting Papers, Society for Economic Dynamics, 103.

Milgrom, P. And N. Stokey (1982): "Information Trade and Common Knowledge," Journal of Economic Theory, 26:1, 17-27.

Milgrom, P. R. (1979): "A Convergence Theorem for Competetive Bidding with Differential Information," Econometrica, 47, 679-688.

Milgrom, P. R. and R. J. Weber (1982): "A Theory of Auctions and Competitive Bidding," Econometrica, 50, 1089-1122.

Ostrovsky, M. (2012): "Information Aggregation in Dynamic Markets with Strategic 
Traders," Econometrica, 80, 2595-2649.

PALfRey, T. R. (1985): "Uncertainty resolution, private information aggregation and the Cournot competitive limit," The review of economic studies, 52, 69-83.

Pesendorfer, W. and J. M. Swinkels (1997): "The Loser's Curse and Information Aggregation in Common Value Auctions," Econometrica, 65(6), 1247-1281.

Rayo, L. And I. Segal (2010): "Optimal Information Disclosure," The Journal of Political Economy, 118, 949-987.

Rostek, M. And M. Weretka (2012): "Price inference in small markets," Econometrica, $80,687-711$.

Siga, L. And M. Minm (2018): "Information Aggregation in Large Markets," Working Paper.

Swinkels, J. M. (1999): "Education Signaling with Preemptive Offers," Review of Economic Studies, 66, 949-970.

Vives, X. (1988): "Aggregation of information in large Cournot markets," Econometrica: Journal of the Econometric Society, 851-876.

Wilson, R. (1977): "A Bidding Model of Perfect Competition," The Review of Economic Studies, 44, 511-518. 


\section{A Proofs for Section 2}

Proof of Property 1. For (i) and (ii), see the proof of Lemma 1 in Daley and Green (2016). Conditional on reaching the second trading period and the buyers' belief, $\pi_{i}$, the strategic setting for trading a given asset $i$ is identical to theirs. Moreover, by their Lemma A.3, the bid price must earn zero expected profit. To demonstrate (iii), we will show that the bid price must be either $v_{L}$ or $c_{H}$ when $\pi_{i}=\bar{\pi}$ by ruling out all other bids.

Clearly, at $t=2$, the reservation price of the low-type seller is $c_{L}$ and the reservation price of the high-type seller is $c_{H}$. Hence, if the bid is strictly above $c_{H}$, both types will accept w.p.1 and the winning buyer earns negative expected profit. Next, suppose there is positive probability that the bid is strictly less than $v_{L}$. Then, for $\epsilon>0$ small enough, a buyer could earn strictly positive expected profit by deviating and offering $v_{L}-\epsilon$. Finally, if the bid is strictly between $v_{L}$ and $c_{H}$, the high type will reject, the low type will accept and the winning buyer makes negative profit. Thus, we have shown that the equilibrium bid price at $t=2$ when $\pi_{i}=\bar{\pi}$ must be either $v_{L}$ or $c_{H}$.

Proof of Property 2. Since $c_{H}>c_{L}$ and $F_{H} \geq F_{L}$, the continuation value of the low type seller from rejecting the bid $v_{L}$ in the first period satisfies:

$$
\begin{aligned}
Q_{L}^{i} & =(1-\delta) \cdot c_{L}+\delta \cdot \mathbb{E}_{L}\left\{F_{L}\left(\pi_{i}, \phi_{i}\right)\right\} \\
& <(1-\delta) \cdot c_{H}+\delta \cdot \mathbb{E}_{L}\left\{F_{L}\left(\pi_{i}, \phi_{i}\right)\right\} \\
& \leq(1-\delta) \cdot c_{H}+\delta \cdot \mathbb{E}_{L}\left\{F_{H}\left(\pi_{i}, \phi_{i}\right)\right\}
\end{aligned}
$$

Therefore, in order to prove that $Q_{H}^{i}>Q_{L}^{i}$, it is sufficient to show that $\mathbb{E}_{H}\left\{F_{H}\left(\pi_{i}, \phi_{i}\right)\right\} \geq$ $\mathbb{E}_{L}\left\{F_{H}\left(\pi_{i}, \phi_{i}\right)\right\}$. Recall that $F_{H}$ is increasing in $\pi_{i}$ and independent of $\phi_{i}$. Hence, the desired inequality is implied by proving that conditional on $\theta_{i}=H$, the random variable $\pi_{i}$ (weakly) first-order stochastically dominates $\pi_{i}$ conditional on $\theta_{i}=L$.

Note that the distribution of $\pi_{i}$ in the second period is a function of the trading probabilities of the seller $i$ and of the realization of news from sellers $j \neq i, z_{i}^{j} \in\{0,1\}$. Fix the interim belief $\pi_{\sigma_{i}}$, and consider news $\mathbf{z}_{-i}^{\prime}$ and $\mathbf{z}_{-i}^{\prime \prime}$ (which occur with positive probability) such that the posterior $\pi_{i}$ satisfies $\pi_{i}\left(\mathbf{z}_{-i}^{\prime}\right) \geq \pi_{i}\left(\mathbf{z}_{-i}^{\prime \prime}\right)$, i.e., $\mathbf{z}_{-i}^{\prime}$ is "better news" for seller $i$ than $\mathbf{z}_{-i}^{\prime \prime}$. But note that:

$$
\frac{\pi_{i}^{I n t} \cdot \rho_{H}^{i}\left(\mathbf{z}_{-i}^{\prime}\right)}{\pi_{i}^{I n t} \cdot \rho_{H}^{i}\left(\mathbf{z}_{-i}^{\prime}\right)+\left(1-\pi_{i}^{I n t}\right) \cdot \rho_{L}^{i}\left(\mathbf{z}_{-i}^{\prime}\right)}=\pi_{i}\left(\mathbf{z}_{-i}^{\prime}\right) \geq \pi_{i}\left(\mathbf{z}_{-i}^{\prime \prime}\right)=\frac{\pi_{i}^{I n t} \cdot \rho_{H}^{i}\left(\mathbf{z}_{-i}^{\prime \prime}\right)}{\pi_{i}^{I n t} \cdot \rho_{H}^{i}\left(\mathbf{z}_{-i}^{\prime \prime}\right)+\left(1-\pi_{i}^{I n t}\right) \cdot \rho_{L}^{i}\left(\mathbf{z}_{-i}^{\prime \prime}\right)}
$$

which implies that $\frac{\rho_{H}\left(\mathbf{z}_{-i}^{\prime}\right)}{\rho_{L}\left(\mathbf{z}_{-i}^{\prime}\right)} \geq \frac{\rho_{H}\left(\mathbf{z}_{-i}^{\prime \prime}\right)}{\rho_{L}\left(\mathbf{z}_{-i}^{\prime \prime}\right)}$, i.e. the ratio of distributions $\frac{\rho_{H}(\cdot)}{\rho_{L}(\cdot)}$ satisfies the monotone likelihood ratio property. This in turn implies that $\rho_{H}(\cdot)$ first-order stochastically dominates $\rho_{H}(\cdot)$, which establishes the result.

Proof of Property [3. We first show that the bid in the first period is $v_{L}$ w.p.1. From Property 2, the strict ranking of seller continuation values implies that, in any equilibrium, if the high type is willing to accept an offer with positive probability then the low type must accept w.p.1. Thus, given Assumption 1, any bid at or above $c_{H}$ would lead to negative expected profit. Any bid in $\left(v_{L}, c_{H}\right)$ also leads to losses since it is only accepted by the low type. If the bid was strictly less than $v_{L}$, a buyer can make strictly positive profits by offering $v_{L}-\epsilon$, for 
$\epsilon>0$ small enough. Thus, any deterministic offer strictly below $v_{L}$ can be ruled out. The only deterministic bid possible is $v_{L}$, at this point there is no profitable deviation for the other buyer than offering $v_{L}$ as well. The same arguments rule out any mixed strategy equilibrium that has a mass point anywhere other than $v_{L}$. Finally, mixing continuously over some interval of offers cannot be an equilibrium. We show this by contradiction. If one of the buyers mixes over some interval $[\underline{b}, \bar{b}]$ with $\bar{b}=v_{L}$ then the other buyer must be offering $v_{L}$ with probability 1 because otherwise he would never want to offer $v_{L}$, which leads to zero profits w.p.1. If instead $\bar{b}<v_{L}$, the other buyer's best response can never have $\underline{b}$ (or anything below) as part of its support. This bid will lose with probability 1 and thus earn zero profits, while bidding $\frac{\bar{b}+v_{L}}{2}$ would lead to strictly positive profits.

Next, it is clear that the high type would reject bid $v_{L}$, since $v_{L}<c_{H}$. To see that the low type must accept with probability less than one, note that if in equilibrium the low type accepted w.p.1, then the posterior belief would assign probability 1 to the type being high in the next period. The offer in the second period (as argued in Property 1) would then be $v_{H}$ but, given Assumption 2, the low type seller would then want to deviate and trade in period 2 at $v_{H}$ rather than at $v_{L}$ in period 1.

Proof of Property 4. The proof that all equilibria involve strictly positive probability of trade in the first period is in the text. We show here that all equilibria must be symmetric. In search of a contradiction, assume there exists an equilibrium in which $\sigma_{A}>\sigma_{B} \geq 0$ for some $A, B \in\{1, \ldots, N\}$. We establish the result by first showing that the beliefs for seller $A$ are more favorable than for seller $B$, following all news realizations; then we show that good news about seller $A$ are more likely to arrive than good news about seller $B$.

Let $\pi_{\sigma_{i}}^{\text {Int }}$ denote the interim belief when (low type) seller $i$ trades w.p. $\sigma_{i}$ (by Property 3 the high type does not trade in first period). Consider the posterior belief about seller $i \in\{A, B\}$ following some news $\mathbf{z}_{-i}=\left(z_{i}^{j}\right)_{j \neq i}$ :

$$
\pi_{i}\left(\mathbf{z}_{-i}\right)=\frac{\pi_{\sigma_{i}}^{I n t} \cdot \rho_{H}^{i}\left(\mathbf{z}_{-i}\right)}{\pi_{\sigma_{i}}^{I n t} \cdot \rho_{H}^{i}\left(\mathbf{z}_{-i}\right)+\left(1-\pi_{\sigma_{i}}^{I n t}\right) \cdot \rho_{L}^{i}\left(\mathbf{z}_{-i}\right)}
$$

where we can express $\rho_{\theta}^{i}\left(\mathbf{z}_{-i}\right)$ as:

$$
\rho_{\theta}^{i}\left(\mathbf{z}_{-i}\right)=\sum_{s \in\{l, h\}} \mathbb{P}\left(S=s \mid \theta_{i}=\theta\right) \cdot \mathbb{P}\left(\left(z_{i}^{j}\right)_{j \neq i, i^{\prime}} \mid S=s\right) \cdot \mathbb{P}\left(z_{i}^{i^{\prime}} \mid S=s\right)
$$

for $i, i^{\prime} \in\{A, B\}$ and $i^{\prime} \neq i$. Note that $\rho_{\theta}^{i}\left(\mathbf{z}_{-i}\right)$ depends on $\sigma_{i^{\prime}}$ only through the term $\mathbb{P}\left(z_{i}^{i^{\prime}} \mid S\right)$. We now show that $\sigma_{A}>\sigma_{B}$ implies that:

$$
\frac{1-\pi_{\sigma_{A}}^{I n t}}{\pi_{\sigma_{A}}^{I n t}} \cdot \frac{\rho_{L}^{A}\left(\mathbf{z}_{-i}\right)}{\rho_{H}^{A}\left(\mathbf{z}_{-i}\right)}<\frac{1-\pi_{\sigma_{B}}^{\text {Int }}}{\pi_{\sigma_{B}}^{I n t}} \cdot \frac{\rho_{L}^{B}\left(\mathbf{z}_{-i}\right)}{\rho_{H}^{B}\left(\mathbf{z}_{-i}\right)},
$$

which will establish that $\pi_{A}\left(\mathbf{z}_{-i}\right)>\pi_{B}\left(\mathbf{z}_{-i}\right)$ for all news $\mathbf{z}_{-i}$. There are two cases to consider, depending on whether $z_{i}^{i^{\prime}}=0$ or $z_{i}^{i^{\prime}}=1$.

If $z_{i}^{i^{\prime}}=1$, then $\mathbb{P}\left(z_{i}^{i^{\prime}}=1 \mid S=s\right)=\sigma_{i^{\prime}} \cdot \mathbb{P}\left(\theta_{i^{\prime}}=L \mid S=s\right)$ and the likelihood ratio $\frac{1-\pi_{\sigma_{i}}^{I n t}}{\pi_{\sigma_{i}}^{I n t}} \cdot \frac{\rho_{L}^{i}\left(\mathbf{z}_{-i}\right)}{\rho_{H}^{i}\left(\mathbf{z}_{-i}\right)}$ 
decreases in $\sigma_{i}$ but is independent of $\sigma_{i^{\prime}}$. Intuitively, if seller $i^{\prime}$ traded, her type is revealed to be low, and the intensity with which she trades is irrelevant for updating. But then inequality (20) follows because $\pi_{\sigma_{i}}^{\text {Int }}$ is increasing in $\sigma_{i}$.

If $z_{i}^{i^{\prime}}=0$, then $\mathbb{P}\left(z_{i}^{i^{\prime}}=0 \mid S=s\right)=1-\sigma_{i^{\prime}} \cdot \mathbb{P}\left(\theta_{i^{\prime}}=L \mid S=s\right)$, and now the likelihood ratio $\frac{1-\pi_{\sigma_{i}}^{I n t}}{\pi_{\sigma_{i}}^{I n t}} \cdot \frac{\rho_{L}^{i}\left(\mathbf{z}_{-i}\right)}{\rho_{H}^{i}\left(\mathbf{z}_{-i}\right)}$ decreases in both $\sigma_{i}$ and $\sigma_{i^{\prime}}$. However, given that both $i$ and $i^{\prime} \operatorname{did}$ not trade (both are good news for $i$ ), inequality (20) follows because the assets $i$ and $i^{\prime}$ are imperfectly correlated and $\frac{1-\pi_{\sigma_{i}}^{I n t}}{\pi_{\sigma_{i}}^{I n t}} \cdot \frac{\rho_{L}^{i}\left(\mathbf{z}_{-i}\right)}{\rho_{H}^{i}\left(\mathbf{z}_{-i}\right)}$ is more sensitive to trading probability $\sigma_{i}$ than to $\sigma_{i^{\prime}}$.

Now, note that $\sigma_{A}>\sigma_{B}$ also implies that the probability that seller $B$ trades and releases bad news about seller $A$ is lower than the probability that seller $A$ trades and releases bad news about seller $B$. Since the posteriors following good news are higher than following bad news, this establishes the result.

Finally, the symmetry in $\phi_{i}$ follows from monotonicity of $Q_{L}^{i}$ in $\phi_{i}$ whenever buyer mixing is part of an equilibrium.

Proof of Proposition 1. To prove existence of an equilibrium, it suffices to show there exists a $(\sigma, \phi) \in[0,1]^{2}$ such that equation (8) holds, i.e., $Q_{L}(\sigma, \phi)=v_{L}$ where the second argument states that all other sellers also trade with intensity $\sigma$. Note that by varying $\sigma$ from 0 to 1 , $Q_{L}$ ranges from $\left[(1-\delta) c_{L}+\delta v_{L},(1-\delta) c_{L}+\delta v_{H}\right]$. By continuity of $Q_{L}$ and Assumption 2, the intermediate value theorem gives the result.

Let us denote by $\pi_{i}\left(\mathrm{z}_{-i} ; \sigma\right)$ the posterior belief following news realization $\mathbf{z}_{-i}$ in an equilibrium with trading probability $\sigma$. Consider the following two candidate equilibria. The first candidate is an equilibrium in which the posterior belief about the seller satisfies $\pi_{i}(\mathbf{z}(0) ; \sigma)=\bar{\pi}$, and the second candidate equilibrium is when the posterior belief about the seller satisfies $\pi_{i}(\mathbf{z}(N) ; \sigma)=$ $\bar{\pi}$. Although there can be other equilibria as well, we do not focus on them. We will now show that these two equilibria coexist when $\lambda$ and $\delta$ are large enough.

1. $\pi_{i}(\mathbf{z}(0) ; \sigma)=\bar{\pi}$. Note that there is at most one such equilibrium since the trading intensity $\sigma$ in this category is fully pinned down by the requirement that $\pi_{i}(\mathbf{z}(0) ; \sigma)=\bar{\pi}$. Let $x$ be the value of $\sigma$ such that $\pi_{i}(\mathbf{z}(0) ; x)=\bar{\pi}$. As $\phi$ varies from 0 and $1, Q_{L}(x, \phi)$ varies continuously from $(1-\delta) c_{L}+\delta v_{L}$ to $(1-\delta) c_{L}+\delta\left(\rho_{L}^{i}(\mathbf{z}(0)) c_{H}+\left(1-\rho_{L}^{i}(\mathbf{z}(0))\right) v_{L}\right)$ where $\rho_{L}^{i}(\mathbf{z}(0))>0$. Hence, there exists a $\delta_{\lambda}^{*}<1$, such that $Q_{L}(x, 1)=v_{L}$. Clearly, this equilibrium exists if $\delta>\delta_{\lambda}^{*}$. Moreover, it is straightforward to show that $\inf _{\lambda} \rho_{L}^{i}(\mathbf{z}(0))>0$. Hence, this equilibrium exists if $\delta$ is larger than $\delta^{*} \equiv \sup _{\lambda \in\left(1-\pi_{0}, 1\right)} \delta_{\lambda}^{*}<1$.

2. $\pi_{i}(\mathbf{z}(N) ; \sigma)=\bar{\pi}$. Note that there is at most one such equilibrium since the trading intensity $\sigma$ is fully pinned down by the requirement that $\pi_{i}(\mathbf{z}(N) ; \sigma)=\bar{\pi}$. Let $y$ be the value of $\sigma$ such that $\pi_{i}(\mathbf{z}(N) ; y)=\bar{\pi}$. As $\phi$ varies from 0 to $1, Q_{L}$ varies continuously from

$$
(1-\delta) c_{L}+\delta\left(\rho_{L}^{i}(\mathbf{z}(N)) v_{L}+\sum_{\mathbf{z}_{-i} \neq \mathbf{z}(N)} \rho_{L}^{i}\left(\mathbf{z}_{-i}\right) V\left(\pi_{i}\left(\mathbf{z}_{-i} ; y\right)\right)\right)
$$

to

$$
(1-\delta) c_{L}+\delta\left(\rho_{L}^{i}(\mathbf{z}(N)) c_{H}+\sum_{\mathbf{z}_{-i} \neq \mathbf{z}(N)} \rho_{L}^{i}\left(\mathbf{z}_{-i}\right) V\left(\pi_{i}\left(\mathbf{z}_{-i} ; y\right)\right)\right)
$$


Moreover, it is straightforward to show that $\lim _{\lambda \rightarrow 1} \rho_{L}^{i}(\mathbf{z}(N))=1$. Therefore, it follows that the range of $Q_{L}$ converges to the interval $\left((1-\delta) c_{L}+\delta v_{L},(1-\delta) c_{L}+\delta c_{H}\right]$ as $\lambda$ goes to 1 . By Assumption 2, $v_{L}$ is in this interval. This establishes the existence of the threshold $\lambda_{\delta}^{*}$ such that this equilibrium exists whenever $\delta>\delta^{*}$ and $\lambda>\lambda_{\delta}^{*}$.

Thus, we conclude that multiple equilibria exist when $\delta>\delta^{*}$ and $\lambda>\lambda_{\delta}^{*}$.

\section{B Proofs for Section 3}

Proof of Lemma 1. Uniqueness of equilibrium follows from the fact that $Q_{L}^{\text {fict }}=(1-\delta) c_{L}+$ $\delta v_{H}>v_{L}$ when $\sigma=1$, and because $Q_{L}^{\text {fict }}$ is monotonically increasing in $\sigma$, and in $\phi$ when buyer mixing is part of an equilibrium. Hence, the unique equilibrium must feature no trade if $Q_{L}^{f i c t}(0,0) \geq v_{L}$. Finally, it is straightforward to check that $Q_{L}^{f i c t}(0,0) \geq v_{L}$ holds if and only if $\lambda \geq \bar{\lambda}$ and $\delta \geq \bar{\delta}$.

For the proof of Theorem 1, it will be useful to reference the following lemma, which is straightforward to verify so the proof is omitted. Let $\pi_{i}(s ; \sigma)$ denote the buyers' posterior belief about seller $i$ following a rejection, conditional on observing that the state is $s$. Then, for $s \in\{l, h\}$, we have:

$$
\pi_{i}(s ; \sigma)=\frac{\pi_{\sigma}^{\text {Int }} \cdot \mathbb{P}\left(S=s \mid \theta_{i}=H\right)}{\pi_{\sigma}^{\text {Int }} \cdot \mathbb{P}\left(S=s \mid \theta_{i}=H\right)+\left(1-\pi_{\sigma}^{\text {Int }}\right) \cdot \mathbb{P}\left(S=s \mid \theta_{i}=L\right)},
$$

where as before $\pi_{\sigma}^{I n t}$ is the interim belief.

Lemma B.1 Given a sequence $\left\{\sigma_{N}\right\}_{N=1}^{\infty}$ of trading probabilities corresponding to a sequence of equilibria along which information aggregates, we also have convergence of posteriors: $\pi_{i}\left(\mathbf{z}_{-i} ; \sigma_{N}\right) \rightarrow^{p}$ $\pi_{i}\left(S ; \sigma_{N}\right)$ as $N \rightarrow \infty$.

Proof of Theorem 1. Part $(i)$. Suppose to the contrary that $\star$ holds with strict inequality, but that information aggregation obtains. Recall that in equilibrium, for any $N$, we must have:

$$
v_{L}=Q_{L}^{i}\left(\sigma_{N}, \phi_{i}\right)=(1-\delta) c_{L}+\delta \sum_{\mathbf{z}_{-i}} \rho_{L}^{i}\left(\mathbf{z}_{-i}\right) \cdot F_{L}\left(\pi_{i}\left(\mathbf{z}_{-i} ; \sigma_{N}\right), \phi_{i}\right)
$$

where

$$
\begin{aligned}
\sum_{\mathbf{z}_{-i}} \rho_{L}^{i}\left(\mathbf{z}_{-i}\right) \cdot F_{L}\left(\pi_{i}\left(\mathbf{z}_{-i} ; \sigma_{N}\right), \phi_{i}\right) & =\sum_{s=l, h} \mathbb{P}\left(S=s \mid \theta_{i}=L\right) \sum_{\mathbf{z}_{-i}} \mathbb{P}\left(\mathbf{z}_{-i} \mid S=s\right) \cdot F_{L}\left(\pi_{i}\left(\mathbf{z}_{-i} ; \sigma_{N}\right), \phi_{i}\right) \\
& >\lambda \cdot v_{L}+(1-\lambda) \cdot \sum_{\mathbf{z}_{-i}} \mathbb{P}\left(\mathbf{z}_{-i} \mid S=h\right) \cdot F_{L}\left(\pi_{i}\left(\mathbf{z}_{-i} ; \sigma_{N}\right), \phi_{i}\right)
\end{aligned}
$$

because in equilibrium we must have $F_{L}\left(\pi_{i}\left(\mathbf{z}(0) ; \sigma_{N}\right), \phi_{i}\right)>v_{L}$.

Since by Lemma B.1, $\pi_{i}\left(\mathbf{z}_{-i} ; \sigma_{N}\right) \rightarrow^{p} \pi_{i}\left(h ; \sigma_{N}\right)$ when the state is $h$, and because ( $\star$ holding strictly implies that $\pi_{i}\left(h ; \sigma_{N}\right)>\pi_{i}(h ; 0)>\bar{\pi}$, we have that for a given $\epsilon>0$, if $N$ is large 
enough, then:

$$
\sum_{\mathbf{z}_{-i}} \mathbb{P}\left(\mathbf{z}_{-i} \mid S=h\right) \cdot F_{L}\left(\pi_{i}\left(\mathbf{z}_{-i} ; \sigma_{N}\right), \phi_{i}\right)>V\left(\pi_{i}\left(h ; \sigma_{N}\right)\right)-\epsilon .
$$

Therefore, we conclude that for sufficiently large $N$ :

$$
\begin{aligned}
v_{L}=Q_{L}^{i}\left(\sigma_{N}, \phi_{i}\right) & >(1-\delta) c_{L}+\delta \cdot\left(\lambda \cdot v_{L}+(1-\lambda) \cdot V\left(\pi_{i}\left(h ; \sigma_{N}\right)\right)\right)-\delta \cdot(1-\lambda) \cdot \epsilon \\
& >(1-\delta) c_{L}+\delta \cdot\left(\lambda \cdot v_{L}+(1-\lambda) \cdot V\left(\pi_{i}(h ; 0)\right)\right)-\delta \cdot(1-\lambda) \cdot \epsilon .
\end{aligned}
$$

Since $\epsilon$ was arbitrary, it must be that:

$$
v_{L} \geq(1-\delta) c_{L}+\delta \cdot\left(\lambda \cdot v_{L}+(1-\lambda) \cdot V\left(\pi_{i}(h ; 0)\right)\right)
$$

which violates $(\star x)$ holding with strict inequality, a contradiction.

Part $(i i)$. If $(\star$ does not hold, then in the fictitious economy, the unique equilibrium trading probability in the first period must satisfy $\sigma^{*}>0$. We next construct an equilibrium sequence $\left\{\sigma_{N}\right\}$ of the actual economy such that the sequence is uniformly bounded away from zero, which then implies that information aggregates along this sequence. First, consider a sequence $\left\{\widehat{\sigma}_{N}\right\}$, not necessarily an equilibrium one, such that $\widehat{\sigma}_{N}=\widehat{\sigma} \in\left(0, \sigma^{*}\right)$, i.e., this is a sequence of constant trading probabilities that are positive but strictly below $\sigma^{*}$. Along such a sequence, information clearly aggregates and, by Lemma B.1. $\pi_{i}\left(\mathbf{z}_{-i}, \widehat{\sigma}_{N}\right) \rightarrow^{p} \pi_{i}\left(S, \widehat{\sigma}_{N}\right)$. Therefore, combined with the fact that $\pi_{i}\left(\mathbf{z}_{-i}, \widehat{\sigma}_{N}\right)=\pi_{i}\left(\mathbf{z}_{-i}, \widehat{\sigma}\right)<\pi_{i}\left(\mathbf{z}_{-i}, \sigma^{*}\right)$, there exists an $N^{*}$ such that for $N>N^{*}$, we have:

$$
\mathbb{E}_{L}\left\{F_{L}\left(\pi_{i}\left(\mathbf{z}_{-i}, \widehat{\sigma}_{N}\right), \phi_{i}\right)\right\}<\mathbb{E}_{L}^{f i c t}\left\{F_{L}\left(\pi_{i}\left(S, \sigma^{*}\right)\right)\right\}=\frac{v_{L}-(1-\delta) \cdot c_{L}}{\delta}
$$

where the last equality holds since $\sigma^{*}>0$ implies that, in the fictitious economy, the low type must be indifferent to trading at $t=1$ and delaying trade to $t=2$. The correspondence $\mathbb{E}_{L}\left\{F_{L}\left(\pi_{i}\left(\mathbf{z}_{-i}, \sigma\right), \cdot\right)\right\}$ is upper hemicontinuous in $\sigma$ for each $N$, and has a maximal value of $v_{H}$ that is strictly greater than $\mathbb{E}_{L}^{f i c t}\left\{F_{L}\left(\pi_{i}\left(S, \sigma^{*}\right)\right)\right\}$. Hence, for each $N>N^{*}$, we can find a $\sigma_{N}$ such that $\sigma_{N} \geq \widehat{\sigma}_{N}>0$ and $\mathbb{E}_{L}\left\{F_{L}\left(\pi_{i}\left(\mathbf{z}_{-i}, \sigma_{N}\right), \phi_{i}\right)\right\}=\frac{v_{L}-(1-\delta) \cdot c_{L}}{\delta}$. This delivers the desired equilibrium sequence $\left\{\sigma_{N}\right\}$ along which information aggregates.

Proof of Theorem 2, We establish the conditions for the coexistence of aggregating and non-aggregating equilibria. To do so, we first show that if $\lambda>\bar{\lambda}$, there exists a $\delta_{2}(\lambda)<1$ such that non-aggregating equilibria exist if $\delta>\delta_{2}(\lambda)$. Second, we show that for $\lambda$ large enough $\delta_{2}(\lambda)<\bar{\delta}_{\lambda}$. Therefore, both non-aggregating and aggregating equilibria exist if $\delta \in\left(\delta_{2}(\lambda), \bar{\delta}_{\lambda}\right)$, since ( $\star$ ) is violated (see Theorem 1).

Consider a candidate sequence of equilibria with trading probabilities $\left\{\sigma_{N}\right\}$, such that $\sigma_{N}=$ $\kappa_{N} \cdot N^{-1}$ and:

$$
\pi_{i}\left(\mathbf{z}(0) ; \kappa_{N} \cdot N^{-1}\right)=\bar{\pi}
$$


Solving (21) for $\kappa_{N}$ and taking the limit as $N \rightarrow \infty$ gives $\kappa_{N} \rightarrow \kappa$ where

$$
\kappa \equiv \frac{1}{\lambda-\frac{(1-\lambda)\left(1-\pi_{0}\right)}{\pi_{0}}} \cdot \log \left(\frac{\lambda-\left(\frac{1-\bar{\pi}}{\bar{\pi}} \cdot \frac{\pi_{0}}{1-\pi_{0}}\right) \cdot \frac{(1-\lambda)\left(1-\pi_{0}\right)}{\pi_{0}}}{\left(\frac{1-\bar{\pi}}{\bar{\pi}} \cdot \frac{\pi_{0}}{1-\pi_{0}}\right) \cdot\left(1-\frac{(1-\lambda)\left(1-\pi_{0}\right)}{\pi_{0}}\right)-(1-\lambda)}\right) \in(0, \infty) .
$$

Seller $i$ expects to receive an offer of $v_{L}$ in all events other than $\mathbf{z}(0)$ and an expected offer $\phi_{i} c_{H}+\left(1-\phi_{i}\right) v_{L}$ for some $\phi_{i} \in[0,1]$ in the event $\mathbf{z}(0)$. Therefore, the sequence of trading probabilities defined above constitutes an equilibrium if $\delta$ is sufficiently high and the probability of the event $\mathbf{z}(0)$ conditional on the seller's type being low is bounded away from zero. To establish the latter, note that:

$$
\begin{aligned}
\mathbb{P}\left(\mathbf{z}(0) \mid \theta_{i}=L\right) & =\sum_{s=l, h} \mathbb{P}\left(S=s \mid \theta_{i}=L\right) \cdot\left(1-\sigma_{N} \cdot \mathbb{P}\left(\theta_{i}=L \mid S=s\right)\right)^{N} \\
& =\sum_{s=l, h} \mathbb{P}\left(S=s \mid \theta_{i}=L\right) \cdot\left(1-\kappa_{N} \cdot N^{-1} \cdot \mathbb{P}\left(\theta_{i}=L \mid S=s\right)\right)^{N} \\
& \rightarrow \sum_{s=l, h} \mathbb{P}\left(S=s \mid \theta_{i}=L\right) \cdot e^{-\kappa \cdot \mathbb{P}\left(\theta_{i}=L \mid S=s\right)}>0
\end{aligned}
$$

where the last limit as $N \rightarrow \infty$ follows from Lemma B.2. In these equilibria, information fails to aggregate because as a result $\mathbb{P}(\mathbf{z}(0) \mid S=s)$ is bounded away from zero in both states of nature (see Lemma B.4). Thus, for each $\lambda>\bar{\lambda}$, we have established the existence of a $\delta_{2}(\lambda)<1$ such that non-aggregating equilibria exist whenever $\delta>\delta_{2}(\lambda)$. Finally, from $(22)$ we have that:

$$
\lim _{\lambda \rightarrow 1} \sum_{s=l, h} \mathbb{P}\left(S=s \mid \theta_{i}=L\right) \cdot e^{-\kappa \cdot \mathbb{P}\left(\theta_{i}=L \mid S=s\right)}=\frac{1-\bar{\pi}}{\bar{\pi}} \cdot \frac{\pi_{0}}{1-\pi_{0}} \in(0,1),
$$

and hence $\lim _{\lambda \rightarrow 1} \delta_{2}(\lambda)<1$. Letting $\underline{\delta}=\lim _{\lambda \rightarrow 1} \delta_{2}(\lambda)$ and noting that $\lim _{\lambda \rightarrow 1} \bar{\delta}=1$ establishes the result.

Next, we establish that when $\lambda<\bar{\lambda}$ or $\delta$ is sufficiently small, then only aggregating equilibria exist. First, suppose that $\lambda<\bar{\lambda}$ and assume to the contrary that information aggregation fails along some sequence of equilibria, and pick a subsequence of equilibria with $\sigma_{N} \rightarrow 0$ as $N$ goes to $\infty$ (See Lemma B.3 for the existence of such a subsequence). But note that for each $N$, we have $\pi_{i}\left(\mathbf{z}_{-i}, \sigma_{N}\right) \leq \pi_{i}\left(h, \sigma_{N}\right)$, i.e., the posterior beliefs must be weakly lower than if the state were revealed to be high. Since $\pi_{i}\left(h, \sigma_{N}\right)$ is continuous in $\sigma_{N}$, and since $\lambda<\bar{\lambda}$ implies that $\pi_{i}(h, 0)<\bar{\pi}$, it follows that for $N$ large enough all posterior beliefs are strictly below $\bar{\pi}$. But then for $N$ large, $Q_{L}^{i}<v_{L}$ and therefore $\sigma_{N}=1$, contradicting Property 3 ,

Second, consider $\dot{\delta}$ defined by $v_{L}=(1-\dot{\delta}) c_{L}+\dot{\delta} V\left(\pi_{0}\right)$, and assume that $\delta<\dot{\delta}$ (Note that Assumption 2 can still be satisfied since $\left.V\left(\pi_{0}\right)<c_{H}\right)$. Suppose to the contrary that information aggregation fails along a sequence of equilibria, and again pick a subsequence of equilibria with $\sigma_{N} \rightarrow 0$ as $N$ goes to $\infty$. By continuity, we must also have that $\pi_{\sigma_{N}}^{I n t} \rightarrow \pi_{0}$ along 
this subsequence. But, note that for each $N$ along this subsequence, it must be that:

$$
\begin{aligned}
v_{L}=Q_{L}^{i}\left(\sigma_{N}, \phi_{i}\right) & =(1-\delta) c_{L}+\delta \mathbb{E}_{L}\left\{F_{L}\left(\pi_{i}\left(\mathbf{z}_{-i}, \sigma_{N}\right), \phi_{i}\right)\right\} \\
& \leq(1-\delta) c_{L}+\delta \mathbb{E}_{L}\left\{V\left(\pi_{i}\left(\mathbf{z}_{-i}, \sigma_{N}\right)\right)\right\} \\
& \leq(1-\delta) c_{L}+\delta V\left(\pi_{\sigma_{N}}^{I n t}\right)
\end{aligned}
$$

where the first inequality follows immediately from $(6)$ and the second from the fact that $V$ is linear function and $\pi_{i}\left(\mathbf{z}_{-i}, \sigma_{N}\right)$ is a supermartingale conditional on $\theta_{i}=L$. Since $\delta<\dot{\delta}$ and $V\left(\pi_{\sigma_{N}}^{I n t}\right) \rightarrow V\left(\pi_{0}\right)$, the last expression is lower than $v_{L}$ for $N$ large enough, a contradiction.

Proof of Proposition 2. Consider a sequence of equilibria along which information aggregates and let $\left\{\sigma_{N}\right\}$ denote the corresponding sequence of trading probabilities. By Theorem 1 , it must be that either $(\star)$ holds with equality or it is violated, i.e. $Q_{L}^{\text {fict }}=v_{L}$ and $\sigma^{\text {fict }} \geq 0$. If $\left\{\sigma_{N}\right\}$ (or any subsequence of it) were strictly above $\sigma^{\text {fict }}$ and bounded away from it, then it is straightforward to show that, for $N$ large enough, due to the convergence of posteriors (see Lemma B.1) we have $Q_{L, N}>Q_{L}^{f i c t}=v_{L}$ and therefore $\sigma_{N}=0$, a contradiction. On the other hand, if $\sigma_{N}$ were strictly below $\sigma^{\text {fict }}$ and bounded away from it, then again, for $N$ large enough, we would have $Q_{L, N}<Q_{L}^{\text {fict }}=v_{L}$ and therefore $\sigma_{N}=1$, a contradiction. Hence, $\sigma_{N} \rightarrow \sigma^{\text {fict }}$.

Conditional on state $s$, the fraction of assets traded in the first period converges to $\sigma^{\text {fict }} \mathbb{P}\left(\theta^{i}=\right.$ $L \mid S=s$ ) and the price is $v_{L}$ for all $N$; in the second period, the buyers' posteriors about the seller converge to $\pi\left(s, \sigma^{\text {fict }}\right)$ (see Lemma B.1), which implies that the prices and the fraction of assets traded converge as well. Thus, conditional on state $S$, the aggregate volatility of prices and of trading volume (in both periods) goes to zero.

Finally, in equilibrium, we have $Q_{L, N}=v_{L}=Q_{L}^{f i c t}$ and the convergence of posteriors implies that $Q_{H, N} \rightarrow Q_{H}^{f i c t}$, which establishes the welfare result.

Proof of Proposition 3. The bounds on the equilibrium trading probabilities follow from Lemma B.3.

Along any convergent subsequence of $\left\{N \sigma_{N}\right\}$, by the Poisson Limit Theorem, the distribution of the number of assets traded in the first period in state $s$ goes to Poisson with some parameter $\kappa \cdot \mathbb{P}\left(\theta^{i}=L \mid S=s\right)$, where $\kappa \in(0, \infty)$. Therefore, even as $N \rightarrow \infty$, conditional on state $s$, the aggregate volatility of trading volume in the first period remains strictly positive. Since this also implies that the buyers' posteriors in the second period remain uncertain, conditional on state $s$, the aggregate volatility of the prices and of trading volume in the second period remains strictly positive as well.

We now prove the welfare result. In order to do so, we will use results from Section 4, where we study the optimal information policy. We will use the fact that the agents' welfare is always weakly greater under optimal information policy than in equilibrium, since the laissez-faire equilibrium is in the planner's choice set.

Consider a sequence of equilibria such that aggregation fails along any of its subsequences. By Theorems 1 and 2, there are two cases to consider: either (1) $\lambda>\bar{\lambda}$ and $\delta>\bar{\delta}$ (i.e. ( $\star$ ) holds with strict inequality) and thus aggregation fails along any sequence of equilibria, or $(2) \lambda>\bar{\lambda}$ and $\delta<\bar{\delta}$ (i.e. $\star$ is violated) and thus there is coexistence of aggregating and non-aggregating equilibria. We ignore the non-generic cases where $\lambda=\bar{\lambda}$ or $\delta=\bar{\delta}$.

Case 1. In equilibrium, $Q_{L, N}\left(\sigma_{N}, \phi_{N}\right)=v_{L}$. By arguments similar to those in the proof of Lemma 3, there is an information policy $G$ with support $\left\{0, \bar{p}\left(\sigma_{N}\right), 1\right\}$ such that $Q_{L}^{G}\left(\sigma_{N}, \phi_{N}\right) \leq$ 
$v_{L}$ and $Q_{H}^{G}\left(\sigma_{N}, \cdot\right) \geq Q_{H, N}\left(\sigma_{N}, \cdot\right)$ where:

$$
Q_{H}^{G}(\sigma, \cdot)=c_{H}+\delta \cdot \mathbb{P}^{G}(1 \mid H) \cdot\left(V(\pi(1 ; \sigma))-c_{H}\right),
$$

where $\mathbb{P}^{G}(1 \mid H)=\mathbb{P}\left(S=h \mid \theta_{i}=H\right) \cdot \frac{\mu_{1}}{\pi_{0}}$, and $\mu_{1}=\mathbb{P}^{G}(1)$. Next, by setting $\phi_{N}=0$ and increasing $\mu_{1}$ such that $Q_{L}^{G}\left(\sigma_{N}, 0\right)=v_{L}$ (possible since $\delta>\bar{\delta}$ ), we ensure that the low type seller is as well off as in equilibrium and the high type seller is strictly better off. Finally, by increasing $\mu_{1}$ to $\pi_{0}$ (thus, fully revealing the state), we further increase $Q_{\theta}^{G}\left(\sigma_{N}, 0\right)$, but $\sigma_{N}$ drops to zero. Since for $N$ large, equilibrium $\sigma_{N}$ is already close to zero, the former effect dominates, implying that $\lim _{N} W_{N}<W^{\text {fict }}$.

Case 2. In equilibrium, again, $Q_{L, N}\left(\sigma_{N}, \phi_{N}\right)=v_{L}$ and, for $N$ large enough, $\sigma_{N}$ must be close to zero and thus strictly below $\sigma^{\text {fict }}>0$. Again, there is an information policy $G$ with support $\left\{0, \bar{p}\left(\sigma_{N}\right), 1\right\}$ such that $Q_{L}^{G}\left(\sigma_{N}, \phi_{N}\right) \leq v_{L}$ and $Q_{H}^{G}\left(\sigma_{N}, \cdot\right) \geq Q_{H, N}\left(\sigma_{N}, \cdot\right)$ where $Q_{H}^{G}$ is as in equation $(23)$. Since $\sigma^{\text {fict }}>\sigma_{N}$ and $\mu_{1} \leq \pi_{0}$, it follows that $Q_{H}^{\text {fict }}\left(\sigma^{\text {fict }}, \cdot\right)>Q_{H}^{G}\left(\sigma_{N}, \cdot\right)$. Thus, for $N$ large enough, the high type seller's payoff is greater in the fictitious economy than in equilibrium, whereas the low type's payoff in both is equal to $v_{L}$. Hence, again we have that $\lim _{N} W_{N}<W^{\text {fict }}$.

Lemma B.2 Let $\left\{\alpha_{x}\right\}$ be any non-negative sequence of real numbers such that $\alpha_{x} \rightarrow \alpha$ as $x \rightarrow \infty$ where $\alpha \in(0,1)$. Then $\left(\frac{x-\alpha_{x}}{x}\right)^{x} \rightarrow e^{-\alpha}$ as $x \rightarrow \infty$.

Proof. Assume that for any $\gamma \in(0,1),\left(\frac{x-\gamma}{x}\right)^{x} \rightarrow e^{-\gamma}$ as $x \rightarrow \infty$. Then, given $\epsilon>0$ so that $\epsilon<\alpha<1-\epsilon$, if $x$ is large enough then $\left|\alpha_{x}-\alpha\right|<\epsilon,\left(\frac{x-\alpha-\epsilon}{x}\right)^{x} \geq e^{-\alpha-\epsilon}-\epsilon$, and $\left(\frac{x-\alpha+\epsilon}{x}\right)^{x} \leq e^{-\alpha+\epsilon}+\epsilon$. This in turn implies that:

$$
e^{-\alpha-\epsilon}-\epsilon \leq\left(\frac{x-\alpha-\epsilon}{x}\right)^{x} \leq\left(\frac{x-\alpha_{x}}{x}\right)^{x} \leq\left(\frac{x-\alpha+\epsilon}{x}\right)^{x} \leq e^{-\alpha+\epsilon}+\epsilon .
$$

Since $\epsilon$ is arbitrary, we conclude that $\left(\frac{x-\alpha_{x}}{x}\right)^{x} \rightarrow e^{-\alpha}$ as $x \rightarrow \infty$. Next, we prove the supposition that for any $\gamma \in(0,1),\left(\frac{x-\gamma}{x}\right)^{x} \rightarrow e^{-\gamma}$ as $x \rightarrow \infty$. Note that $\left(\frac{x-\gamma}{x}\right)^{x}=e^{x \cdot \log \left(\frac{x-\gamma}{x}\right)}$ and by L'Hospital's rule:

$$
\lim _{x \rightarrow \infty} x \cdot \log \left(\frac{x-\gamma}{x}\right)=\lim _{x \rightarrow \infty} \frac{\log \left(\frac{x-\gamma}{x}\right)}{x^{-1}}=-\lim _{x \rightarrow \infty} \frac{\gamma \cdot x}{x-\gamma}=-\gamma .
$$

By continuity, $\lim _{x \rightarrow \infty} e^{x \cdot \log \left(\frac{x-\gamma}{x}\right)}=e^{-\gamma}$.

Lemma B.3 Suppose that there is a sequence of equilibria $\left\{\sigma_{N}\right\}$ along which information aggregation fails. Then there exist a subsequence of equilibria with trading probabilities $\left\{\sigma_{N_{m}}\right\}$ such that for some $0<\underline{\kappa}<\bar{\kappa}<\infty$, we have $\underline{\kappa}<\sigma_{N_{m}} N_{m}<\bar{\kappa}$ for all $m$.

Proof. Suppose for contradiction that for all subsequences with trading probabilities $\left\{\sigma_{N_{m}}\right\}$ we have $\lim _{m \rightarrow \infty} \sigma_{N_{m}} N_{m}=\infty$. Let $X_{i}$ denote the indicator that takes value of 1 if seller $i$ has traded in the first period. Define $Y_{N_{m}}=N_{m}^{-1} \cdot \sum_{i=1}^{N_{m}} X_{i}$ be the fraction of sellers who have traded in the first period, and note that conditional on the state being $s, Y_{N_{m}}$ has a mean $p_{s, N_{m}}$ 
and variance $N_{m}^{-1} \cdot p_{s, N_{m}} \cdot\left(1-p_{s, N_{m}}\right)$, where recall that $p_{s, N_{m}}=\sigma_{N_{m}} \cdot \mathbb{P}\left(\theta_{i}=L \mid S=s\right)$. Since $p_{l, N_{m}}>p_{h, N_{m}}$

$$
\begin{aligned}
\mathbb{P}\left(Y_{N_{m}} \geq \frac{p_{h, N_{m}}+p_{l, N_{m}}}{2} \mid S=h\right) & =\mathbb{P}\left(Y_{N_{m}}-p_{h, N_{m}} \geq \frac{p_{l, N_{m}}-p_{h, N_{m}}}{2} \mid S=h\right) \\
& \leq \mathbb{P}\left(\left(Y_{N_{m}}-p_{h, N_{m}}\right)^{2} \geq\left(\frac{p_{l, N_{m}}-p_{h, N_{m}}}{2}\right)^{2} \mid S=h\right)
\end{aligned}
$$

And by Markov's inequality:

$$
\begin{aligned}
\mathbb{P}\left(\left(Y_{N_{m}}-p_{h, N_{m}}\right)^{2} \geq\left(\frac{p_{l, N_{m}}-p_{h, N_{m}}}{2}\right)^{2} \mid S=h\right) & \leq \frac{\mathbb{E}\left\{\left(Y_{N_{m}}-p_{h, N_{m}}\right)^{2} \mid S=h\right\}}{\left(\frac{p_{l, N_{m}}-p_{h, N_{m}}}{2}\right)^{2}} \\
& =\frac{N_{m}^{-1} \cdot p_{h, N_{m}} \cdot\left(1-p_{h, N_{m}}\right)}{\left(\frac{p_{l, N_{m}}-p_{h, N_{m}}}{2}\right)^{2}} \\
& =4 \cdot \frac{\sigma_{N_{m}} \cdot \mathbb{P}\left(\theta_{i}=L \mid S=h\right)-\sigma_{N_{m}}^{2} \cdot \mathbb{P}\left(\theta_{i}=L \mid S=h\right)^{2}}{N_{m} \cdot \sigma_{N_{m}}^{2} \cdot\left(\mathbb{P}\left(\theta_{i}=L \mid S=l\right)-\mathbb{P}\left(\theta_{i}=L \mid S=h\right)\right)^{2}}
\end{aligned}
$$

which by our assumption tends to 0 as $m \rightarrow \infty$. By a similar reasoning, we have that:

$$
\begin{aligned}
\mathbb{P}\left(Y_{N_{m}}<\frac{p_{h, N_{m}}+p_{l, N_{m}}}{2} \mid S=l\right) & =\mathbb{P}\left(p_{l, N_{m}}-Y_{N_{m}}>\frac{p_{l, N_{m}}-p_{h, N_{m}}}{2} \mid S=l\right) \\
& \leq \mathbb{P}\left(\left(p_{l, N_{m}}-Y_{N_{m}}\right)^{2}>\left(\frac{p_{l, N_{m}}-p_{h, N_{m}}}{2}\right)^{2} \mid S=l\right) \\
& \leq \frac{\mathbb{E}\left\{\left(Y_{N_{m}}-p_{l, N_{m}}\right)^{2} \mid S=l\right\}}{\left(\frac{p_{l, N_{m}}-p_{h, N_{m}}}{2}\right)^{2}} \\
& =\frac{N_{m}^{-1} \cdot p_{l, N_{m}} \cdot\left(1-p_{l, N_{m}}\right)}{\left(\frac{p_{l, N_{m}}-p_{h, N_{m}}}{2}\right)^{2}} \\
& =4 \cdot \frac{\sigma_{N_{m}} \cdot \mathbb{P}\left(\theta_{i}=L \mid S=l\right)-\sigma_{N_{m}}^{2} \cdot \mathbb{P}\left(\theta_{i}=L \mid S=l\right)^{2}}{N_{m} \cdot \sigma_{N_{m}}^{2} \cdot\left(\mathbb{P}\left(\theta_{i}=L \mid S=l\right)-\mathbb{P}\left(\theta_{i}=L \mid S=h\right)\right)^{2}}
\end{aligned}
$$

which again tends to 0 as $m \rightarrow \infty$. Combining these two observations, we conclude that information about the state must aggregate along all subsequences, a contradiction.

Next, suppose for contradiction that for all subsequences with trading probabilities $\left\{\sigma_{N_{m}}\right\}$ we have that $\lim _{m \rightarrow \infty} \sigma_{N_{m}} N_{m}=0$. Then, given any $\epsilon>0$ and $m$ large enough, we have:

$$
\left(1-\sigma_{N_{m}} \cdot \mathbb{P}\left(\theta_{i}=L \mid S=s\right)\right)^{N_{m}}=\left(\frac{N_{m}-\sigma_{N_{m}} \cdot N_{m} \cdot \mathbb{P}\left(\theta_{i}=L \mid S=s\right)}{N_{m}}\right)^{N_{m}} \geq\left(\frac{N_{m}-\epsilon}{N_{m}}\right)^{N_{m}}
$$

for $s \in\{l, h\}$, where the last expression converges to $e^{-\epsilon}$ by Lemma B.2. Since $\epsilon$ is arbitrary, 
$\left(1-\sigma_{N_{m}} \cdot \mathbb{P}\left(\theta_{i}=L \mid S=s\right)\right)^{N_{m}}$ goes to 1 as $m \rightarrow \infty$. Hence, we have that for $\theta \in\{L, H\}:$

$$
\mathbb{P}\left(Y_{N_{m}}=0 \mid \theta_{i}=\theta\right)=\sum_{s=l, h} \mathbb{P}\left(S=s \mid \theta_{i}=\theta\right) \cdot\left(1-\sigma_{N_{m}} \cdot \mathbb{P}\left(\theta_{i}=L \mid S=s\right)\right)^{N_{m}} \rightarrow 1
$$

Now, consider the posterior belief about the seller conditional on event that no seller has traded. For any $m$, since the low type must expect offers above $v_{L}$ with positive probability and since $\mathbf{z}(0)$ is the best possible news, it must be that:

$$
\begin{aligned}
\pi_{i}\left(\mathbf{z}(0), \sigma_{N_{m}}\right) \geq \bar{\pi} & \\
& \Longleftrightarrow \frac{\pi_{\sigma_{N_{m}}}^{\text {Int }} \cdot \mathbb{P}\left(Y_{N_{m}}=0 \mid \theta_{i}=H\right)}{\pi_{\sigma_{N_{m}}}^{\text {Int }} \cdot \mathbb{P}\left(Y_{N_{m}}=0 \mid \theta_{i}=H\right)+\left(1-\pi_{\sigma_{N_{m}}}^{\text {Int }}\right) \cdot \mathbb{P}\left(Y_{N_{m}}=0 \mid \theta_{i}=L\right)} \geq \bar{\pi} .
\end{aligned}
$$

But note that, since $\sigma_{N_{m}} \rightarrow 0$ and $\pi_{\sigma_{N_{m}}}$ is continuous, the left-hand side converges to $\pi<\bar{\pi}$, a contradiction.

Lemma B.4 Consider a sequence of equilibria with trading probabilities $\left\{\sigma_{N}\right\}$ such that $\sigma_{N} N<$ $\bar{\kappa}$ for some $\bar{\kappa}<\infty$. Then $\mathbb{P}\left(Y_{N}=0 \mid S=s\right)$ is bounded away from zero, uniformly over $N$, for $s \in\{l, h\}$.

Proof. We have that $\mathbb{P}\left(Y_{N}=0 \mid S=s\right)=\left(1-p_{s, N}\right)^{N}$ for $s \in\{l, h\}$. By assumption, $p_{s, N} \leq$ $N^{-1} \cdot \bar{\kappa} \cdot \mathbb{P}\left(\theta_{i}=L \mid S=s\right)$. Therefore,

$$
\mathbb{P}\left(Y_{N}=0 \mid S=s\right) \geq\left(1-N^{-1} \cdot \bar{\kappa} \cdot \mathbb{P}\left(\theta_{i}=L \mid S=s\right)\right)^{N}
$$

and by Lemma B.2. $\lim _{N \rightarrow \infty}\left(1-N^{-1} \cdot \bar{\kappa} \cdot \mathbb{P}\left(\theta_{i}=L \mid S=s\right)\right)^{N}=e^{-\bar{\kappa} \cdot \mathbb{P}\left(\theta_{i}=L \mid S=s\right)}>0$.

\section{Proofs for Section 4}

Notation and Preliminaries. Let $g(p)$ denote the probability of posterior $p$ under the information policy $G{ }^{11}$ Let $g_{s}(p)$ denote the probability of posterior $p$ conditional on the state $s$, so that

$$
g(p)=\pi_{0} \cdot g_{h}(p)+\left(1-\pi_{0}\right) \cdot g_{l}(p),
$$

Observe that for any $p$ in the support of $G$, Bayesian consistency requires that

$$
p=\frac{\pi_{0} \cdot g_{h}(p)}{\pi_{0} \cdot g_{h}(p \mid h)+\left(1-\pi_{0}\right) \cdot g_{l}(p)},
$$

Given $g$, (24) and (25) pin down the conditional probabilities $g_{h}$ and $g_{l}$. Let $\mathbb{P}^{G}(p \mid \theta)$ denote the probability that the posterior about the state is $p$ conditional on the seller's type $\theta$ :

$$
\mathbb{P}^{G}(p \mid \theta)=\mathbb{P}(S=h \mid \theta) g_{h}(p)+\mathbb{P}(S=l \mid \theta) g_{l}(p)
$$

\footnotetext{
${ }^{11}$ To streamline notation, we restrict attention to information policies with finite support. Our proofs can easily extended to rule out policies that assign positive mass to a continuum of beliefs not in the support stated in Lemma 3 .
} 
Given any $\sigma$, and $p$ in the support of $G$, the buyers' posterior belief that the seller is type $H$ can be computed as follows:

$$
\pi_{i}(p ; \sigma) \equiv \frac{\pi_{\sigma}^{I n t} \cdot \mathbb{P}^{G}(p \mid H)}{\pi_{\sigma}^{I n t} \cdot \mathbb{P}^{G}(p \mid H)+\left(1-\pi_{\sigma}^{I n t}\right) \cdot \mathbb{P}^{G}(p \mid L)},
$$

where recall that the interim belief is given by $\pi_{\sigma}^{I n t}=\frac{\pi_{0}}{\pi_{0}+\left(1-\pi_{0}\right)(1-\sigma)}$. The continuation value of the $\theta$-type seller, under information policy $G$, is

$$
Q_{\theta}^{G}(\sigma, \phi)=(1-\delta) c_{\theta}+\delta \sum_{p} F_{\theta}\left(\pi_{i}(p ; \sigma), \phi\right) \mathbb{P}^{G}(p \mid \theta)
$$

where $F_{\theta}$ is the payoff function defined by the equations (5) and (6). Recall that $(G, \sigma, \phi)$ is feasible only if $Q_{L}^{G}(\sigma, \phi)$ satisfies (13) and 14 .

Proof of Lemma 2. If $\sigma<\underline{\sigma}$, then $\pi(1 ; \sigma)<\bar{\pi}$ and thus the buyers' bid is $v_{L}$ in the second period w.p.1. But then, $Q_{L}^{G}<v_{L}$, violating (13). If $\sigma>\bar{\sigma}$, then $\pi_{i}(0 ; \sigma)>\bar{\pi}$ and thus the buyers' bid is greater than $c_{H}$ in the second period w.p.1. But then, $Q_{L}^{G}>v_{L}$ (by Assumption 2) and (14) requires $\sigma=0$, a contradiction.

Proof of Lemma 3. First, it is without loss of generality to assume that the optimal policy does not involve posteriors in the interval $(0, \bar{p}(\sigma))$. To see this, note that if the planner were to reassign the weight from any posteriors in $(0, \bar{p}(\sigma))$ to the posteriors in $\{0, \bar{p}(\sigma)\}$ (respecting Bayesian plausibility), she could reduce $\phi$ to ensure that the low type's continuation value is unaffected. Since this change does not affect the distribution of posteriors above $\bar{p}(\sigma)$, the high type's continuation value (and thus welfare) is also unaffected.

Second, suppose that the planner's policy has posteriors in the interval $(\bar{p}(\sigma), 1)$ in its support. We will demonstrate that such a policy can be (weakly) improved upon. There are two cases to consider, depending on whether $(13)$ is slack.

Case 1. Suppose that (13) holds with equality, and thus $Q_{L}^{G}=v_{L}$ and $\sigma \geq 0$. First, we will argue that if the planner were to reassign weight from the posteriors in $(\bar{p}(\sigma), 1)$ to posteriors in $\{\bar{p}(\sigma), 1\}$, then she would reduce (increase) the low (high) type's continuation value. To see this, consider a candidate information policy $G$ that assigns a positive weight to some posterior $p^{\prime} \in(\bar{p}(\sigma), 1)$. Next, consider an alternative policy $\widehat{G}$ that is the same as $G$ except that it reassigns the weight from $p^{\prime}$ to the posteriors $\bar{p}(\sigma)$ and 1 to preserve Bayesian plausiblity, i.e.,

$$
\begin{aligned}
g\left(p^{\prime}\right) & =\widehat{g}(\bar{p}(\sigma))-g(\bar{p}(\sigma))+\widehat{g}(1)-g(1), \\
g\left(p^{\prime}\right) \cdot p^{\prime} & =\mu(\bar{p}(\sigma)) \cdot \bar{p}(\sigma)+\mu(1) \cdot 1 .
\end{aligned}
$$

Define $\mu^{\widehat{G}}(p) \equiv \widehat{g}(p)-g(p)$. By 26$)$, we have:

$$
\underbrace{\mathbb{P}^{G}\left(p^{\prime} \mid \theta\right)}_{\equiv \mu_{\theta}^{G}\left(p^{\prime}\right)}=\underbrace{\mathbb{P}^{\widehat{G}}(\bar{p}(\sigma) \mid \theta)-\mathbb{P}^{G}(\bar{p}(\sigma) \mid \theta)}_{\equiv \mu_{\theta}^{\widehat{G}}(\bar{p}(\sigma))}+\underbrace{\mathbb{P}^{\widehat{G}}(1 \mid \theta)-\mathbb{P}^{G}(1 \mid \theta)}_{\equiv \mu_{\theta}^{\widehat{G}}(1)} .
$$


Next, observe that:

$$
\begin{aligned}
& \mu_{L}^{\widehat{G}}(\bar{p}(\sigma)) V\left(\pi_{i}(\bar{p}(\sigma), \sigma)\right)+\mu_{L}^{\widehat{G}}(1) V\left(\pi_{i}(1, \sigma)\right)<\mu_{L}^{G}\left(p^{\prime}\right) V\left(\pi_{i}\left(p^{\prime}, \sigma\right)\right) \\
& \Longleftrightarrow \mu_{L}^{\widehat{G}}(\bar{p}(\sigma)) \pi_{i}(\bar{p}(\sigma), \sigma)+\mu_{L}^{\widehat{G}}(1) \pi_{i}(1, \sigma)<\mu_{L}^{G}\left(p^{\prime}\right) \pi_{i}\left(p^{\prime}, \sigma\right) \\
& \Longleftrightarrow \frac{\mu_{L}^{\widehat{G}}(\bar{p}(\sigma))}{\mu_{L}^{\widehat{G}}(\bar{p}(\sigma))+\mu_{L}^{\widehat{G}}(1)} \pi_{i}(\bar{p}(\sigma), \sigma)+\left(1-\frac{\mu_{L}^{\widehat{G}}(\bar{p}(\sigma))}{\mu_{L}^{\widehat{G}}(\bar{p}(\sigma))+\mu_{L}^{\widehat{G}}(1)}\right) \pi_{i}(1, \sigma)<\pi_{i}\left(p^{\prime}, \sigma\right),
\end{aligned}
$$

where the last inequality follows from $\frac{\mu_{L}^{\widehat{G}}(\bar{p}(\sigma))}{\mu_{L}^{\widehat{G}}(\bar{p}(\sigma))+\mu_{L}^{\widehat{G}}(1)}>\frac{\mu^{\widehat{G}}(\bar{p}(\sigma))}{\mu^{\widehat{G}}(\bar{p}(\sigma))+\mu^{\widehat{G}}(1)}, \pi_{i}(\bar{p}(\sigma), \sigma)<\pi_{i}\left(p^{\prime}, \sigma\right)<$ $\pi_{i}(1, \sigma)$, and the fact that

$$
\frac{\mu^{\widehat{G}}(\bar{p}(\sigma))}{\mu^{\widehat{G}}(\bar{p}(\sigma))+\mu^{\widehat{G}}(1)} \pi_{i}(\bar{p}(\sigma), \sigma)+\left(1-\frac{\mu^{\widehat{G}}(\bar{p}(\sigma))}{\mu^{\widehat{G}}(\bar{p}(\sigma))+\mu^{\widehat{G}}(1)}\right) \pi_{i}(1, \sigma)=\pi_{i}\left(p^{\prime}, \sigma\right) .
$$

Since the bid following any posterior $p>\bar{p}(\sigma)$ must be pooling (Property 1), it follows that $Q_{L}^{\widehat{G}}(\sigma, \phi)<Q_{L}^{G}(\sigma, \phi)$. By an analogous argument, we can show that:

$$
\mu_{H}^{\widehat{G}}(\bar{p}(\sigma)) V\left(\pi_{i}(\bar{p}(\sigma), \sigma)\right)+\mu_{H}^{\widehat{G}}(1) V\left(\pi_{i}(1, \sigma)\right)>\mu_{H}^{G}\left(p^{\prime}\right) V\left(\pi_{i}\left(p^{\prime}, \sigma\right)\right)
$$

and, therefore, $Q_{H}^{\widehat{G}}(\sigma, \phi)>Q_{H}^{G}(\sigma, \phi)$. Finally, the planner can increase $\sigma$ and/or $\phi$ to some $\widehat{\sigma}$ and $\widehat{\phi}$ to ensure that that $Q_{L}^{\widehat{G}}(\widehat{\sigma}, \widehat{\phi})=v_{L}$, and note that both those adjustments increase the high type's continuation value from $Q^{\widehat{G}}(\sigma, \phi)$ to $Q_{H}^{\widehat{G}}(\widehat{\sigma}, \widehat{\phi})$. Thus, $(\widehat{G}, \widehat{\sigma}, \widehat{\phi})$ generates the same payoff to the low type, but strictly higher payoff for the high type than $(G, \sigma, \phi)$.

Case 2. Suppose that (13) is slack, and thus $Q_{L}^{G}(\sigma, \phi)>v_{L}$ and $\sigma=0$. By inspecting the planner's objective, we see that it must be that $\phi=1$; otherwise the planner could implement a higher $\phi$ and improve welfare without violating (13). Thus, the planner's objective is proportional to $\mathbb{P}\left(\pi \geq \bar{\pi} \mid \theta_{i}=H\right)=\mathbb{P}\left(\tilde{p} \geq \bar{p}(0) \mid \theta_{i}=H\right)$ (see (11)).

Given the properties just established, we will now construct an alternative policy that is feasible and increases the planner's objective, which completes the proof for Case 2. Recall the supposition we are trying to refute is that the optimal policy assigns weight to posteriors in $(\bar{p}(0), 1)$. The alternative policy $G^{\prime}$ reassigns the weight from all the posteriors in $[\bar{p}(0), 1]$ to the posteriors $\{0, \hat{p}\}$, where $\hat{p} \equiv \mathbb{E}_{G}\{\tilde{p} \mid \tilde{p} \geq \bar{p}(0)\}-\varepsilon$ for some $\varepsilon$ small (respecting Bayesian plausibility). If $\varepsilon=0$, then $\hat{p}>\bar{p}(0)$, the trading surplus is unchanged and the low type's continuation value increases (see argument in Case 1), implying that (13) remains slack. For $\varepsilon$ sufficiently small but positive, again $\hat{p}>\bar{p}(0)$ and $(13)$ remains slack by continuity, but the trading surplus strictly increases as Bayesian plausibility implies $\mathbb{P}^{G^{\prime}}\left(\tilde{p}=\hat{p} \mid \theta_{i}=H\right)>\mathbb{P}^{G}(\tilde{p} \geq$ $\left.\bar{p}(0) \mid \theta_{i}=H\right)$.

Proof of Lemma 4. If (13) is required to hold with equality, then the low type's payoff is $v_{L}$ and thus maximization of expected discounted gains from trade is equivalent to maximization of the high type's payoff $Q_{H}^{G}(\sigma, \phi)$. By Lemma 3, we have that:

$$
Q_{H}^{G}(\sigma, \phi)=(1-\delta) c_{H}+\delta\left(c_{H}+\mathbb{P}^{G}(1 \mid H) \cdot\left(V\left(\pi_{i}(h ; \sigma)\right)-c_{H}\right)\right)
$$


where $\mathbb{P}^{G}(1 \mid H)=\mathbb{P}\left(S=h \mid \theta^{i}=H\right) \cdot \frac{\mu_{1}}{\pi_{0}}$. Thus, the planner's objective is increasing in $\mu_{1}$. $\left(V\left(\pi_{i}(h ; \sigma)\right)-c_{H}\right)$, which in turn is increasing in $\mu_{1}$ and in $\sigma$. There are two possibilities.

If $Q_{L}^{\text {fict }}(\bar{\sigma}, 0) \leq v_{L}$, then it is feasible for the planner to set $\mu_{1}=\pi_{0}$ and $\sigma=\bar{\sigma}$, and then choose $\phi$ so that $Q_{L}^{\text {fict }}(\bar{\sigma}, \phi)=v_{L}$. This is clearly optimal since both $\mu_{1}$ and $\sigma$ are maximized subject to feasibility.

If $Q_{L}^{\text {fict }}(\bar{\sigma}, 0)>v_{L}$, then $\mu_{1}=\pi_{0}$ and $\sigma=\bar{\sigma}$ is not feasible, since then (13) would hold with strict inequality; thus, either $\mu_{1}<\pi_{0}, \sigma<\bar{\sigma}$, or both. Note that the low type's continuation value is given by:

$$
Q_{L}^{G}(\sigma, \phi)=(1-\delta) c_{L}+\delta\left(v_{L}+\mathbb{P}^{G}(\bar{p}(\sigma) \mid L) \phi\left(c_{H}-v_{L}\right)+\mathbb{P}^{G}(1 \mid L)\left(V\left(\pi_{i}(h ; \sigma)\right)-v_{L}\right)\right)
$$

where $\mathbb{P}^{G}(1 \mid L)=\mathbb{P}\left(S=h \mid \theta^{i}=L\right) \cdot \frac{\mu_{1}}{\pi_{0}}$.

We now argue that $\phi=0$. To see this, note from (35) that $Q_{L}^{G}(\sigma, 0)$ is continuous in $\mu_{1}$ and $\sigma$ for $\sigma \in[\underline{\sigma}, \bar{\sigma}]$, and $Q_{L}^{G}(\bar{\sigma}, 0)=Q_{L}^{f i c t}(\bar{\sigma}, 0)>v_{L}$ when $\mu_{1}=\pi_{0}$. Thus, if $\phi$ is strictly positive, the planner could reduce $\phi$ to zero, increase $\mu_{1}$ and/or $\sigma$, and still maintain $Q_{L}^{G}=v_{L}$. But this would increase the trading surplus as $Q_{H}^{G}$ is independent of $\phi$, but is increasing in $\mu_{1}$ and $\sigma$.

Therefore, at the informed planner's solution, we have $Q_{L}^{G}(\sigma, 0)=v_{L}$, which is equivalent to saying that $\mu_{1}\left(V\left(\pi_{i}(h ; \sigma)\right)-v_{L}\right)$ is equal to some constant $K>0$, which implies that $\mu_{1}=\frac{K}{V\left(\pi_{i}(h ; \sigma)\right)-v_{L}}$. Substituting for $\mu_{1}$ in $Q_{H}^{G}(\sigma, 0)$, we have:

$$
Q_{H}^{G}(\sigma, 0)=(1-\delta) c_{H}+\delta\left(c_{H}+\mathbb{P}\left(S=h \mid \theta^{i}=H\right) \cdot \frac{K}{\pi_{0}} \cdot \frac{V\left(\pi_{i}(h ; \sigma)\right)-c_{H}}{V\left(\pi_{i}(h ; \sigma)\right)-v_{L}}\right),
$$

which is increasing in $\sigma$. Thus, it is optimal for the planner to increase $\sigma$ as much as possible (by reducing $\left.\mu_{1}\right)$. Since $\mu_{1}+\left(1-\mu_{0}-\mu_{1}\right) \bar{p}(\sigma)=\pi_{0}$, it follows that the lowest $\mu_{1}$ is equal to $\frac{\pi_{0}-\bar{p}(\sigma)}{1-\bar{p}(\sigma)}$, i.e., the planner sets $\mu_{0}=0$. Therefore, the solution to the planner's problem features $\mu_{1}^{*}=\frac{\pi_{0}-\bar{p}\left(\sigma^{*}\right)}{1-\bar{p}\left(\sigma^{*}\right)}$ and $\sigma^{*}$ is such that $Q_{L}^{G}\left(\sigma^{*}, 0\right)=v_{L}$.

Proof of Proposition 4. Let $G^{*}$ denote the solution to the variant of the informed planner's problem characterized in Lemma 4 in which the inequality 13 is required to hold with equality. It is immediate that either (1) the solution to the original informed planner's problem involves (13) holding with strict inequality, or (2) $G^{*}$ is the solution to that problem. Therefore, it is sufficient to compare the value of the planner's objective in these two scenarios.

For (1), in Lemma C.1 we show that the informed planner's solution must have $\sigma=0, \phi=1$, and an information policy, denoted by $G_{0}$, which consists of only two posteriors $\{0, \bar{p}(0)\}$, where by Bayes' rule:

$$
\mathbb{P}^{G_{0}}(\bar{p}(0) \mid \theta)=\mathbb{P}\left(S=h \mid \theta_{i}=\theta\right)+\mathbb{P}\left(S=l \mid \theta_{i}=\theta\right)\left(\frac{1-\bar{p}(0)}{\bar{p}(0)}\right)\left(\frac{\pi_{0}}{1-\pi_{0}}\right) .
$$

The trading surplus under $(G, \sigma, \phi)=\left(G_{0}, 0,1\right)$ is given by:

$$
W^{G_{0}}=\delta\left(\left(1-\pi_{0}\right)\left(v_{L}-c_{L}\right)+\pi_{0} \mathbb{P}^{G_{0}}(\bar{p}(0) \mid H)\left(v_{H}-c_{H}\right)\right) .
$$


For $(2)$, the trading surplus under $(G, \sigma, \phi)=\left(G^{*}, \sigma^{*}, \phi^{*}\right)$ is given by:

$$
\begin{aligned}
W^{G^{*}} & =\left(1-\pi_{0}\right) \cdot\left(Q_{L}^{G^{*}}-c_{L}\right)+\pi_{0} \cdot\left(Q_{H}^{G^{*}}-c_{H}\right) \\
& =\left(1-\pi_{0}\right) \cdot\left(v_{L}-c_{L}\right)+\pi_{0} \cdot \delta \cdot \mathbb{P}^{G^{*}}(1 \mid H) \cdot\left(V\left(\pi_{i}\left(1 ; \sigma^{*}\right)\right)-c_{H}\right),
\end{aligned}
$$

where $\mathbb{P}^{G^{*}}(1 \mid H)=\mathbb{P}(S=h \mid H) \cdot \frac{\mu_{1}^{*}}{\pi_{0}}$ and $\left(\mu_{1}^{*}, \sigma^{*}\right)$ are given in Lemma 4 .

We will establish the result by showing that (i) $W^{G_{0}}<W^{G^{*}}$ when $\delta$ is sufficiently small, (ii) $W^{G_{0}}>W^{G^{*}}$ when $\delta$ is sufficiently large, and (iii) $W^{G_{0}}$ increases faster in $\delta$ than $W^{G^{*}}$.

For (i), a necessary condition for $G_{0}$ to be preferable to $G^{*}$ is that (13) holds with strict inequality when $(G, \sigma, \phi)=\left(G_{0}, 0,1\right)$ :

$$
v_{L}<Q_{L}^{G_{0}}(0,1)=(1-\delta) c_{L}+\delta \cdot\left(v_{L}+\mathbb{P}^{G_{0}}(\bar{p}(0) \mid L) \cdot\left(c_{H}-v_{L}\right)\right),
$$

which is equivalent to requiring that $\delta>\delta_{0}$, where:

$$
\delta_{0} \equiv \frac{v_{L}-c_{L}}{v_{L}-c_{L}+\mathbb{P}^{G_{0}}(\bar{p}(0) \mid L) \cdot\left(c_{H}-v_{L}\right)} .
$$

Otherwise, either (13) is violated so that $\left(G_{0}, 0,1\right)$ is inconsistent with equilibrium, or (13) holds with equality implying $\left(G_{0}, 0,1\right)$ is sub-optimal (see Lemma 4 ). Since $W^{G_{0}}$ and $W^{G^{*}}$ are continuous in $\delta$, it follows that $W^{G^{*}}>W^{G_{0}}$ for $\delta$ close to $\delta_{0}$.

For (ii), note that $(G, \sigma, \phi)=\left(G_{0}, 0,1\right)$ would be optimal if $\delta$ were equal to 1 , since then only the gains from trade in the second period matter for the trading surplus (see Lemma C.1). Since $W^{G_{0}}$ and $W^{G^{*}}$ are continuous in $\delta$, it follows that $W^{G^{*}}<W^{G_{0}}$ for $\delta$ close to 1 .

For (iii), for all $\delta \in\left(\delta_{0}, 1\right)$, we have:

$$
\begin{aligned}
\frac{d W^{G_{0}}}{d \delta} & =\left(1-\pi_{0}\right) \cdot\left(v_{L}-c_{L}\right)+\pi_{0} \cdot \mathbb{P}^{G_{0}}(\bar{p}(0) \mid H) \cdot\left(v_{H}-c_{H}\right) \\
& >\pi_{0} \cdot \mathbb{P}(S=h \mid H) \cdot\left(v_{H}-c_{H}\right)
\end{aligned}
$$

since $\mathbb{P}^{G_{0}}(\bar{p}(0) \mid H)>\mathbb{P}^{G_{0}}(S=h \mid H)$. On the other hand, using the same arguments as in the proof of Lemma 4 , we have that $\mu_{1}^{*} \cdot\left(V\left(\pi_{i}\left(1 ; \sigma^{*}\right)\right)-c_{H}\right)$ is decreasing in $\delta$ and thus

$$
\frac{d W^{G^{*}}}{d \delta}<\pi_{0} \cdot \mathbb{P}(S=h \mid H) \cdot\left(v_{H}-c_{H}\right)
$$

since $\mu_{1}^{*} \leq \pi_{0}$ and $V\left(\pi_{i}\left(1 ; \sigma^{*}\right)\right)<v_{H}$. Thus, $\frac{d W^{G_{0}}}{d \delta}>\frac{d W^{G^{*}}}{d \delta}$ for $\delta \in\left(\delta_{0}, 1\right)$.

Proof of Lemma 5. If $\sigma_{N}=0$ for some $N$, the planner's information policy would be uninformative. Hence, in the second period, the buyers' posterior would equal their prior, which is strictly below $\bar{\pi}$ (Assumption 1 ) and, thus, their bid would equal to $v_{L}$ w.p.1. But this would imply that $Q_{L}^{\mathcal{M}_{N}}(0, \cdot)<v_{L}$, a contradiction.

Proof of Proposition 5. Consider an information policy $\mathcal{M}_{N}$ that consists of a binary message (signal), where the message is denoted by $\omega_{N} \in\{b, g\}$. When the planner observes that the fraction of assets that have traded is weakly greater than $\tau$, she always sends message $\omega_{N}=b$. Otherwise, she sends message $\omega_{N}=g$ w.p. $q$ and message $\omega_{N}=b$ w.p. $1-q$. 
Let us set $q=\mu_{1}^{*}$ and $\tau=\frac{1}{2} \cdot \sigma^{*} \cdot\left(\mathbb{P}\left(\theta_{i}=L \mid S=h\right)+\mathbb{P}\left(\theta_{i}=L \mid S=l\right)\right)$, where $\left(\mu_{1}^{*}, \sigma^{*}\right)$ are given in Lemma 4. By Lemma 5, an equilibrium requires that:

$$
v_{L}=Q_{L}^{\mathcal{M}_{N}}\left(\sigma_{N}, \phi_{N}\right)=(1-\delta) c_{L}+\delta \sum_{\omega_{N}=b, g} \mathbb{P}^{\mathcal{M}_{N}}\left(\omega_{N} \mid L\right) \cdot F_{L}\left(\pi_{i}\left(\omega_{N} ; \sigma_{N}\right), \phi_{N}\right) .
$$

where $\mathbb{P}^{\mathcal{M}_{N}}(\cdot \mid \theta)$ denotes the probability distribution over the planner's message, given the seller's type; and $\pi_{i}\left(\omega_{N} ; \sigma_{N}\right)$ is the posterior about seller $i$ given the planner's message $\omega_{N}$. Since in equilibrium we must have $\pi_{i}\left(g ; \sigma_{N}\right) \geq \bar{\pi} \geq \pi_{i}\left(b ; \sigma_{N}\right)$ (by Assumption 2), the continuation value of the high type seller is given by:

$$
Q_{H}^{\mathcal{M}_{N}}\left(\sigma_{N}, \phi_{N}\right)=c_{H}+\delta \cdot \mathbb{P}^{\mathcal{M}_{N}}(g \mid H) \cdot\left(V\left(\pi_{i}\left(g ; \sigma_{N}\right)\right)-c_{H}\right) .
$$

Next, conjecture that there is an equilibrium sequence $\left\{\sigma_{N}\right\}$ such that:

$$
\mathbb{P}\left(\theta_{i}=L \mid S=h\right) \cdot \sigma_{N}<\tau-\varepsilon<\tau+\varepsilon<\mathbb{P}\left(\theta_{i}=L \mid S=l\right) \cdot \sigma_{N}
$$

for some small $\varepsilon>0$ and $N$ large enough. Clearly, along any such sequence, $\mathbb{P}^{\mathcal{M}_{N}}(g \mid S=h) \rightarrow$ $\mu_{1}^{*}$ and $\mathbb{P}^{\mathcal{M}_{N}}(g \mid S=l) \rightarrow 0$ as $N$ grows large and, as a result, also $\pi_{i}\left(g ; \sigma_{N}\right) \rightarrow \pi_{i}\left(1 ; \sigma_{N}\right)$, where $\pi_{i}\left(1 ; \sigma_{N}\right)$ is the posterior about the seller given that the state is revealed to be $h$. But, since also $Q_{L}^{G^{*}}\left(\sigma^{*}, \phi^{*}\right)=v_{L}$, where $\left(G^{*}, \sigma^{*}, \phi^{*}\right)$ are as in Lemma 4 , by continuity it follows that $\sigma_{N} \rightarrow \sigma^{*}$. Note that, by definition of $\tau$, when $\varepsilon$ is small, we have that:

$$
\mathbb{P}\left(\theta_{i}=L \mid S=h\right) \cdot \sigma^{*}<\tau-\varepsilon<\tau+\varepsilon<\mathbb{P}\left(\theta_{i}=L \mid S=l\right) \cdot \sigma^{*},
$$

and our conjecture is verified. Finally, it is clear that $Q_{H}^{\mathcal{M}_{N}}\left(\sigma_{N}, \phi_{N}\right) \rightarrow Q_{H}^{G^{*}}\left(\sigma^{*}, \phi^{*}\right)$, where the latter is the high type's payoff under the information policy in Lemma 4. This implies convergence of the trading surplus (the low type's payoff is $v_{L}$ ).

Proof of Corollary 1. Follows directly from our characterization of the optimal information policy.

Proof of Corollary 2. Follows directly from our characterization of the optimal information policy, together with the fact that:

$$
Q_{L}^{f i c t}(\bar{\sigma}, 0)=(1-\delta) c_{L}+\delta\left(v_{L}+(1-\lambda) \cdot \pi_{i}(1, \bar{\sigma}) \cdot\left(v_{H}-v_{L}\right)\right)
$$

with $\bar{\sigma}$ as defined in Lemma 2 .

Lemma C.1 Suppose that at the solution to the informed planner's problem (13) holds with strict inequality. Then, the solution must have $\sigma=0, \phi=1$ and an information policy that only consists posteriors $\tilde{p} \in\{0, \bar{p}(0)\}$.

Proof. First, if (13) holds with strict inequality, then $\sigma=0$ by (14). Second, if (13) holds with strict inequality for some $\phi<1$, it must also hold with strict inequality for $\phi=1$ as $Q_{L}^{G}(0, \phi)$ is increasing in $\phi$. By inspection of the planner's objective, we see that the trading surplus is increasing in $\phi$. Thus, it must be that $\phi=1$. 
Given the above observations, the planner's objective reduces to choosing the information policy $G$ in order to maximize $\mathbb{P}^{G}(\tilde{p} \geq \bar{p}(0) \mid H)$. We prove the last claim by way of contradiction. Suppose that the optimal information policy, $G$, consists of posteriors outside $\{0, \bar{p}(0)\}$.

- If there are posteriors in $(0, \bar{p}(0))$, then consider the alternative policy $G^{\prime}$ that reassigns the weight from all such posteriors to the posteriors 0 and $\bar{p}(0)$ (respecting Bayesian plausibility). Note that $Q_{L}^{G^{\prime}}(0,1)>Q_{L}^{G}(0,1)$ since the bid is $v_{L}$ for any $p<\bar{p}(0)$, which ensures that $(13)$ is satisfied under $G^{\prime}$, and it implies that $\mathbb{P}^{G^{\prime}}(\tilde{p} \geq \bar{p}(0) \mid H)>$ $\mathbb{P}^{G}(\tilde{p} \geq \bar{p}(0) \mid H)$ by Bayesian plausibility, which contradicts the optimality of $G$.

- If there are posteriors above $\bar{p}(0)$, then consider the alternative policy $G^{\prime}$, which reassigns the weight from all the posteriors in $[\bar{p}(0), 1]$ to the posteriors 0 and $\hat{p} \equiv \mathbb{E}_{G}\{\tilde{p} \mid \tilde{p} \geq \bar{p}(0)\}-\varepsilon$ (respecting Bayesian plausibility). Using the same arguments as in Case 2 in the proof of Lemma 3, if $\varepsilon$ is small enough, then (13) continues to be satisfied while trading surplus strictly increases, which again contradicts the optimality of $G$.

\section{Infinite Horizon Model}

In this section, we extend our aggregation results to a setting with an infinite number of trading opportunities $t \in\{1,2, \ldots\}$. Intuitively, one might expect that with more trading periods there are more opportunities to learn from trading behavior and hence more information will be revealed. However, there is a countervailing force; there are more opportunities for (strategic) sellers to signal through delay. It turns out that two factors essentially cancel each other out.

Besides allowing for an infinite number of trading opportunities, the model and the information structure is identical to the one presented in Section 2. The only additional notation we will require is the public history at (the end of) date $t$, which we denote by $\mathbf{z}^{t}=\left\{\mathbf{z}_{1}, \ldots, \mathbf{z}_{t}\right\}$, consists of the history of all the trades that have taken place at dates prior to and including $t$. Note that $\mathbf{z}^{t}$ also corresponds to buyers' information set prior to making offers in date $t+1$.

Characterizing the set of all possible equilibria in the infinite horizon model is more difficult because the space of relevant histories is a complex object. In principle, the path of play can depend on sellers' beliefs about the quality of other sellers' assets, the distribution of buyers' beliefs about the quality of each seller's asset, the buyers' and the sellers' beliefs about the aggregate state, as well as the number of assets remaining on the market. Nevertheless, we are able to obtain sharp predictions regarding the information aggregation properties of the set of equilibria.

In order to illustrate these findings, we must generalize our notion of information aggregation. Let $\pi_{t, N}^{\text {State }}$ denote the buyers' posterior belief that the state is high, conditional on having observed the trading history, $\mathbf{z}^{t}$, in an economy with $N+1$ sellers.

Definition 5 There is information aggregation at date $\boldsymbol{t}$ along a given sequence of equilibria if $\pi_{t, N}^{\text {State }} \rightarrow^{p} 1_{\{S=h\}}$ as $N \rightarrow \infty$. 
We say that information aggregates along a given sequence if there exists a $t<\infty$ such that information aggregates at date $t$. Otherwise, we say that information aggregation fails.

The following theorem shows that, with an infinite trading horizon, $(\star x)$ is indeed necessary and sufficient to rule out aggregating equilibria.

Theorem 3 Consider the infinite horizon model.

(i) If (太) holds with strict inequality, then information aggregation fails along any sequence of equilibria.

(ii) If ( ) does not hold, then there exists a sequence of equilibria along which information aggregates.

(iii) There exists a $\underline{\delta}<1$ such that whenever $\delta \in(\underline{\delta}, \bar{\delta})$ and $\lambda$ is sufficiently large, there is coexistence of sequences of equilibria along which information aggregates with sequences of equilibria along which aggregation fails.

The proof hinges on arguments similar to those used in the two-period economy. For $(i)$, we show that the earliest date in which information about the state is supposed to aggregate is similar to the first period in a two-period economy. That is, suppose that information aggregates at some date $\tau$ but not before. Because ( $\star$ ) holds, the option value of waiting for the state to be revealed is sufficiently high to make sellers strictly prefer to delay trade at date $\tau$. But if sellers do not trade in date $\tau$, then no information is revealed, which means that $\tau$ cannot possibly be the earliest date of aggregation.

In order to establish $(i i)$ and (iii), we construct a class of equilibria that essentially share the information aggregation properties of the two-period economy. A feature of this class is that once the belief about the seller weakly exceeds $\bar{\pi}$, all future bids are pooling. When $(\star x)$ does not hold (i.e., $\delta<\bar{\delta}$ or $\lambda<\bar{\lambda}$ ), we show that such equilibria exist and that there is an equilibrium sequence within this class along which information aggregates. Then, following arguments similar to those for the proof of Theorem 2, we show that under the conditions stated in (iii), there also exists another sequence of equilibria (still within the class) in which aggregation fails.

\section{Proof of Theorem 3}

We establish parts (i)-(iii) of Theorem 3 separately.

Proof of Theorem 3, part (i). We proceed by contradiction and suppose to the contrary that there is some finite date $t$ at which information aggregates. In particular, suppose that information has not aggregated before $t$, but it aggregates at $t$. Consider seller $i$ who trades with probability in $(0,1)$ at $t$. We know that the number of such sellers must grow to $\infty$ with $N$, since otherwise there would be insufficient information learned at $t$. Without loss of generality assume that all sellers trade with probability in $(0,1)$ at $t$. By the skimming property, the bid for this seller's asset must be $v_{L}$, which the high type rejects whereas the low type accepts with some probability $\sigma_{i, N} \in(0,1)$.

Let $Q_{L, t}^{i, N}$ denote the low type seller $i$ 's continuation value from rejecting a bid $v_{L}$ at time $t$. Define

$$
\bar{Q}_{t}^{N} \equiv(1-\delta) \cdot c_{L}+\delta \cdot\left(\lambda_{L, t} \cdot v_{L}+\left(1-\lambda_{L, t}\right) \cdot V\left(\pi_{i}(h ; 0)\right)\right.
$$


where (i) $\lambda_{L, t}=\mathbb{P}_{t}\left(S=l \mid \theta_{i}=L\right)$ is the posterior belief that the state is $l$ conditional on trading history up to period $t$ and the seller's type being $L$, and (ii) $\pi_{i}(h ; 0)$ is the posterior belief about the seller $i$ conditional on the state being $h$. In Lemma D.1, we show that:

$$
\lim _{N \rightarrow \infty} \mathbb{P}_{t}\left(Q_{L, t}^{i, N} \geq \bar{Q}_{t}^{N}\right)=1
$$

i.e., $\bar{Q}_{t}^{N}$ provides a lower bound on the low type's continuation value. Next, we use this result to show that with probability bounded away from zero in both states of nature, if $N$ is large enough, then $Q_{L, t}^{i, N}>v_{L}$. This immediately implies that the low types strictly prefer to delay trade at $t$, contradicting aggregation and thus establishing our result.

Since $\star \star$ holds, $\bar{Q}_{1}^{N}>v_{L}$. Thus, information aggregation must fail in the first period. In LemmaD.2, we show that failure of information aggregation at $t$ implies that the probability of the event that no seller trades in that period must be bounded away from zero, uniformly over $N$, in both states of nature. Because this event is 'good' news about the state, then following it in the first period, we have $\lambda_{L, 2}<\lambda_{L, 1}$ and, thus, $\bar{Q}_{2}^{N}>v_{L}$. But then again information aggregation must fail in the second period and, therefore, the probability that no seller trades in the second period must remain bounded away from zero in both states of nature. Repeating this argument until period $t$, we can construct a history that occurs with probability bounded away from zero in both states of nature, in which $\bar{Q}_{t}^{N}>v_{L}$, as was stated above.

Proof of Theorem 3, part (ii). The proof is by construction. Consider a candidate equilibrium in which for any period $t$, the following properties hold:

(i) If $\pi_{i, t}<\bar{\pi}$, then the bid is $v_{L}$, which the low type accepts w.p. $\sigma_{t} \in[0,1)$ whereas the high type rejects w.p.1.

(ii) If $\pi_{i, t}>\bar{\pi}$, then the bid is $V\left(\pi_{i, t}\right)$ and both types accept it w.p.1.

(iii) If $\pi_{i, t}=\bar{\pi}$, then the bid is $V\left(\pi_{i, t}\right)$ w.p. $\phi_{t}$ (and both types accept it w.p.1) and is $v_{L}$ w.p. $1-\phi_{t}$ (and both types reject it).

The only off-equilibrium path event in a candidate satisfying (i)-(iii) is a rejection when $\pi_{i, t}>\bar{\pi}$, in which case the interim belief as given by Bayes rule is not well defined. For such cases, we specify $\pi_{\sigma_{i}, t}=\pi_{i, t}$ (i.e., unexpected rejections are attributed to random trembles).12

We will now verify that an equilibrium satisfying (i)-(iii) exists (with off-path beliefs as specified immediately above). To do so, consider any history and let $N_{t}\left(\mathcal{N}_{t}\right)$ denote the number (set) of sellers who have not yet traded at the beginning of period $t$. Notice that the seller's value function under the proposed equilibrium is the same as in (5) and (6), where $\left(\pi_{i}, \phi_{i}\right)$ is replaced by $\left(\pi_{i, t}, \phi_{t}\right)$. By symmetry of the candidate, $\pi_{i, t}=\pi_{j, t}=\pi_{t}$ for all $j \neq i \in \mathcal{N}_{t}$. We now show that there exists $\left(\sigma_{t}, \phi_{t+1}\right)$ such that profitable deviations do not exist:

(i) Suppose that $\pi_{t}<\bar{\pi}$. Since continuation values in period $t+1$ are the same as in the two-period model, we know by Proposition 1, that for any $N_{t}$, there exists at least one $\left(\sigma_{t}, \phi_{t+1}\right)$ pair such that the low types' continuation value is exactly $v_{L}$. Hence, a low-type seller is willing to mix. Clearly, a high-type seller strictly prefers to reject.

\footnotetext{
${ }^{12}$ The precise specification of off-path beliefs is not crucial for the construction, any $\pi_{\sigma_{i}, t} \leq \min \left\{g\left(\pi_{i, t}\right), 1\right\}$ will suffice, where $g\left(\pi_{i, t}\right)>\pi_{i, t}$ is such that $(1-\delta) c_{H}+\delta V\left(g\left(\pi_{i, t}\right)\right)=V\left(\pi_{i, t}\right)$ and ensures that a high type cannot profitably deviate from rejecting.
} 
(ii) Suppose that $\pi_{t}>\bar{\pi}$ and seller $i$ rejects, Since all other seller accept w.p.1. there is no information revealed by other sellers and therefore (given the off-path specification above) $\pi_{i, t+1}=\pi_{t}$. Therefore, rejecting the offer leads to a payoff of $(1-\delta) c_{\theta}+\delta F_{\theta}\left(\pi_{t}\right)<V\left(\pi_{t}\right)$.

(iii) Suppose that $\pi_{t}=\bar{\pi}$. If trade does not occur, buyers in period $t+1$ attribute all rejections to a low offer made by buyers in period $t$. Hence, if the seller rejects, $\pi_{i, t+1}=\pi_{i, t}$ and by the same argument as in (ii), such a deviation is not profitable for the seller.

That buyers do not have a profitable deviation from the candidate follows a similar reasoning to the argument for Property 1 in the two-period model. Belief consistency is by construction. Thus, there exists an equilibrium of the candidate form.

Notice that, by construction, the second period payoff to a type- $\theta$ seller is the same as in the two-period model. Therefore, if $(\star)$ does not hold then following the same argument as in the proof of Theorem 1, we can construct a sequence of first-period trading probabilities $\left\{\sigma_{N, 1}\right\}$ that are uniformly bounded away from zero, which ensures information aggregates in the first period.

Proof of Theorem 3, part (iii). From Theorem 3, part (ii), when ( $\star$ ) does not hold, there is a sequence of equilibria along which information aggregates. In the class of equilibria constructed in the proof of Theorem 3 (ii), equilibrium play in the first play coincides with the equilibrium play of all equilibria in the two period economy of Section 3. As a result, under the same conditions as in Theorem 2 (namely, that $\delta \in(\underline{\delta}, \bar{\delta})$ ), there exists a sequence of equilibria along which information aggregation fails in the first period. Furthermore, by construction of this sequence of equilibria (see proof of Theorem 2), (i) the probability of the event that no seller trades in the first period remains bounded away from zero in both states of nature and (ii) the posterior belief about the seller in the second period following this event is equal to $\bar{\pi}$. But then, following this event, by construction no additional information about the state is revealed through trade.

In what follows, we prove the two lemmas used in the proof of Theorem 3 , part (i).

Lemma D.1 Suppose that ( $\star$ ) holds, and information aggregates in period $t$ but not before. Then $\lim _{N \rightarrow \infty} \mathbb{P}_{t}\left(Q_{L, t}^{i, N} \geq \bar{Q}_{t}^{N}\right)=1$.

Proof. The low type's continuation value from rejecting bid $v_{L}$ at date $t$ is:

$$
\begin{aligned}
Q_{L, t}^{i, N} & =(1-\delta) \cdot c_{L}+\delta \cdot\left(\lambda_{L, t} \cdot \mathbb{E}_{t}\left\{F_{L, t+1}^{i, N} \mid S=l\right\}+\left(1-\lambda_{L, t}\right) \cdot \mathbb{E}_{t}\left\{F_{L, t+1}^{i, N} \mid S=h\right\}\right) \\
& >(1-\delta) \cdot c_{L}+\delta \cdot\left(\lambda_{L, t} \cdot v_{L}+\left(1-\lambda_{L, t}\right) \cdot \mathbb{E}_{t}\left\{F_{L, t+1}^{i, N} \mid S=h\right\}\right),
\end{aligned}
$$

where $\mathbb{E}_{t}\left\{F_{L, t+1}^{i, N} \mid S=s\right\}$ denotes the low type's expected payoff conditional on history up to $t$ and the state being $s$. For the inequality, we used the fact that the payoffs at $t+1$ must be strictly above $v_{L}$ with positive probability, since otherwise no seller would be willing to delay trade to $t+1$. We next show that, for any $\varepsilon>0$,

$$
\lim _{N \rightarrow \infty} \mathbb{P}_{t}\left(F_{L, t+1}^{i, N} \geq V\left(\pi_{i}(h ; 0)\right)-\varepsilon \mid S=h\right)=1,
$$


which, since $\varepsilon$ is arbitrary, will establish the result.

Suppose that the state is $h$ and let $T$ be the smallest number of periods such that:

$$
\left(1-\delta^{T}\right) \cdot c_{H}+\delta^{T} \cdot v_{H}<V\left(\pi_{i}(h ; 0)\right)
$$

which is finite since $\star$ implies $V\left(\pi_{i}(h ; 0)\right)>c_{H}$. Since information aggregates at $t$ (by hypothesis), we can choose $N$ large enough so that (w.p. close to 1 ) the agents' belief that the state is $h$ is close to 1 in the periods $t+1$ through $t+1+T$. Let us consider histories in which this is the case. If we show that (w.p. going to 1 as $N$ goes to $\infty$ ) the bid at $t+1$ is pooling and both seller types accept the bid, then we are done.

Suppose to the contrary that for any $N$, there is strictly positive probability (bounded away from zero) that the bid is not pooling at $t+1$. There are two cases to consider at $t$. First, it could be that, with probability bounded away from zero, the buyers make a bid that is rejected by both types. Second, it could be that, with probability bounded away from zero, the bid is $v_{L}$ and the low types accept it with positive probability.

The first case is straightforward to rule out, since otherwise the buyers could profitably deviate and attract both seller types to trade at date $t$. For the second case, note that also at $t+2$, with probability bounded away from zero, the bid $v_{L}$ must be made and accepted by the low type with some probability. Otherwise, if the pooling bid were made instead (w.p. close to 1 ), the low type would not be willing to trade at $t+1$ (Assumption 2). We can repeat this argument until and including period $T$ and construct sub-histories that occur with probability bounded away from zero, in which the buyers make a bid $v_{L}$ which is accepted with positive probability by the low types in periods $t+1$ through $t+1+T$.

Let $\Omega_{\tau}$ denote the set of sub-histories at $\tau \in\{t+1, \ldots t+1+T\}$ in which the bid is $v_{L}$ in periods $t+1$ through $\tau$, and let $\omega_{\tau}$ denote an element of $\Omega_{\tau}$. For $\tau^{\prime}>\tau$, let $\Omega_{\tau^{\prime}} \mid \omega_{\tau}$ denote the sub-histories in $\Omega_{\tau^{\prime}}$ that have $\omega_{\tau}$ as a predecessor. Now, for any $\omega_{\tau} \in \Omega_{\tau}$, in order for buyers not to be able to attract the high type at $\tau$, it must be that:

$$
V\left(\pi_{i, \tau}\left(\omega_{\tau}\right)\right) \leq Q_{H, \tau}^{i, N}\left(\omega_{\tau}\right)
$$

i.e., the high type would weakly prefer to reject a pooling offer and get his continuation value. The high type's continuation value in turn satisfies:

$$
Q_{H, \tau}^{i, N}\left(\omega_{\tau}\right) \leq(1-\delta) \cdot c_{H}+\delta \cdot \max \left\{\mathbb{E}_{H}\left\{Q_{H, \tau+1}^{i, N} \mid\left\{\Omega_{\tau+1} \mid \omega_{\tau}\right\}\right\}, \mathbb{E}_{H}\left\{V\left(\pi_{i, \tau+1}\right) \mid\left\{\Omega_{\tau+1} \mid \omega_{\tau}\right\}^{c}\right\}\right\} .
$$

Since the beliefs that the state is $h$ are arbitrarily close to 1 in all periods $\tau \in\{t+1, t+1+T\}$, the posterior beliefs about the seller are arbitrarily close to each other in any such period $\tau$. Hence, combining with (53), for any $\varepsilon>0$, we can choose $N$ large enough so that:

$$
Q_{H, \tau}^{i, N}\left(\omega_{\tau}\right) \leq(1-\delta) \cdot c_{H}+\delta \cdot \mathbb{E}_{H}\left\{Q_{H, \tau+1}^{i, N} \mid\left\{\Omega_{\tau+1} \mid \omega_{\tau}\right\}\right\}+\varepsilon
$$

for all $\tau \in\{t+1, t+1+T\}$, which implies that:

$$
Q_{H, t+1}^{i, N}\left(\omega_{t+1}\right) \leq\left(1-\delta^{T}\right) \cdot c_{H}+\delta^{T} \cdot \mathbb{E}_{H}\left\{Q_{H, t+1+T}^{i, N} \mid\left\{\Omega_{t+1+T} \mid \omega_{\tau+1}\right\}\right\}+\hat{\varepsilon}
$$

where $\hat{\varepsilon}$ can be made small by choosing $\varepsilon$ small. Since the value to the seller in any period 
cannot exceed $v_{H}$, then with probability approaching 1 as $N$ goes to $\infty$,

$$
V\left(\pi_{i}(h ; 0)\right)-\hat{\varepsilon} \leq V\left(\pi_{i, t+1}\left(\omega_{t+1}\right)\right) \leq\left(1-\delta^{T}\right) \cdot c_{H}+\delta^{T} \cdot v_{H}+\hat{\varepsilon},
$$

which, since $\hat{\varepsilon}$ is arbitrary, contradicts our choice of $T$.

Lemma D.2 Suppose that information aggregation fails at $t$ along a sequence of equilibria with time-t trading probabilities $\left\{\sigma_{i, N}\right\}$. Then there is a subsequence of equilibria along which the probability that no seller trades at $t$ remains bounded away from zero, in both states of nature.

Proof. Assume that there is a subsequence of equilibria with trading probabilities $\left\{\sigma_{N_{m}}\right\}$ with $\sum_{i=1}^{N_{m}} \sigma_{i, N_{m}}<\bar{\kappa}<\infty$ for some $\bar{\kappa}>0$ and all $m$. Note that $1-\sigma_{i, N_{m}} \cdot P\left(\theta_{i}=L \mid S=s\right) \geq e^{-\sigma_{i, N_{m}} \cdot K}$ for any $K$ satisfying $1-P\left(\theta_{i}=L \mid S=l\right) \geq e^{-K}$. But for any such $K$, we have:

$$
\begin{aligned}
P(\text { no seller trades at } t \mid S=s) & =\Pi_{i=1}^{N_{m}}\left(1-\sigma_{i, N_{m}} \cdot P\left(\theta_{i}=L \mid S=s\right)\right) \\
& \geq \Pi_{i=1}^{N_{m}} e^{-\sigma_{i, N_{m}} \cdot K} \\
& =e^{-K \cdot \sum_{i=1}^{N_{m}} \sigma_{i, N_{m}}} \\
& \geq e^{-K \cdot \bar{\kappa}}>0
\end{aligned}
$$

which establishes the result.

We are left to prove the assertion that there is a subsequence $\left\{\sigma_{N_{m}}\right\}$ with $\sum_{i=1}^{N_{m}} \sigma_{i, N_{m}}<$ $\bar{\kappa}<\infty$ for some $\bar{\kappa}>0$ and all $m$. Suppose to the contrary that for all subsequences $\lim _{m \rightarrow \infty} \sum_{i=1}^{N_{m}} \sigma_{i, N_{m}}=\infty$. Let $X_{i} \in\{0,1\}$ denote the indicator that seller $i$ has traded and $Y_{N_{m}}=N_{m}^{-1} \sum_{i=1}^{N_{m}} X_{i}$ denote the fraction of sellers who have traded. Let $p_{i, N_{m}}(s)=$ $\sigma_{i, N_{m}} \cdot \mathbb{P}\left(\theta_{i}=L \mid S=s\right)$ and note that:

$$
\mu_{N_{m}}(s) \equiv \mathbb{E}\left\{Y_{N_{m}} \mid S=s\right\}=N_{m}^{-1} \cdot \sum_{i=1}^{N_{m}} p_{i, N_{m}}(s)
$$

and

$$
\nu_{N_{m}}(s) \equiv \mathbb{E}\left\{\left(Y_{N_{m}}-\mu_{N_{m}}(s)\right)^{2} \mid S=s\right\}=N_{m}^{-2} \cdot \sum_{i=1}^{N_{m}} p_{i, N_{m}}(s) \cdot\left(1-p_{i, N_{m}}(s)\right) .
$$

Since $\mu_{N_{m}}(l)>\mu_{N_{m}}(h)$,

$$
\begin{aligned}
P\left(Y_{N_{m}} \geq \frac{\mu_{N_{m}}(h)+\mu_{N}(l)}{2} \mid S=h\right) & =P\left(Y_{N_{m}}-\mu_{N_{m}}(h) \geq \frac{\mu_{N_{m}}(l)-\mu_{N_{m}}(h)}{2} \mid S=h\right) \\
& \leq P\left(\left(Y_{N_{m}}-\mu_{N_{m}}(h)\right)^{2} \geq\left(\frac{\mu_{N_{m}}(l)-\mu_{N_{m}}(h)}{2}\right)^{2} \mid S=h\right)
\end{aligned}
$$


And, by Markov's inequality:

$$
\begin{array}{r}
P\left(\left(Y_{N_{m}}-\mu_{N_{m}}(h)\right)^{2} \geq\left(\frac{\mu_{N_{m}}(l)-\mu_{N_{m}}(h)}{2}\right)^{2} \mid S=h\right) \leq \frac{\nu_{N_{m}}(h)}{\left(\frac{\mu_{N_{m}}(l)-\mu_{N_{m}}(h)}{2}\right)^{2}} \\
=\frac{N_{m}^{-2} \cdot \sum_{i=1}^{N_{m}} p_{i, N_{m}}(h) \cdot\left(1-p_{i, N_{m}}(h)\right)}{\left(N_{m}^{-1} \cdot \sum_{i=1}^{N_{m}} \frac{p_{i, N_{m}}(l)-p_{i, N_{m}}(h)}{2}\right)^{2}} \\
=4 \cdot \frac{\sum_{i=1}^{N_{m}} \sigma_{i, N_{m}} \cdot P\left(\theta_{i}=L \mid S=h\right)-\sum_{i=1}^{N_{m}} \sigma_{i, N_{m}}^{2} \cdot P\left(\theta_{i}=L \mid S=h\right)^{2}}{\left(\sum_{i=1}^{N_{m}} \sigma_{i, N_{m}}\right)^{2} \cdot\left(P\left(\theta_{i}=L \mid S=l\right)-P\left(\theta_{i}=L \mid S=h\right)\right)^{2}},
\end{array}
$$

which by our assumption tends to 0 as $m$ goes to $\infty$. By a similar reasoning, we have that:

$$
\mathbb{P}\left(Y_{N_{m}}<N_{m}^{-1} \cdot \sum_{i=1}^{N_{m}} \frac{p_{i, N_{m}}(l)+p_{i, N_{m}}(h)}{2} \mid S=l\right) \rightarrow 0,
$$

as $m$ goes to $\infty$. Combining these two observations, we conclude that information about the state must aggregate along all subsequences, a contradiction. 